\title{
Matter wave explorer of gravity (MWXG)
}

W. Ertmer • C. Schubert • T. Wendrich • M. Gilowski • M. Zaiser •

T. v. Zoest • E. Rasel • Ch. J. Bordé - A. Clairon · Landragin •

P. Laurent • P. Lemonde • G. Santarelli • W. Schleich • F. S. Cataliotti •

M. Inguscio - N. Poli • F. Sorrentino • C. Modugno • G. M. Tino • P. Gill •

H. Klein • H. Margolis • S. Reynaud • C. Salomon • A. Lambrecht • E. Peik •

C. Jentsch • U. Johann • A. Rathke • P. Bouyer · L. Cacciapuoti ·

P. De Natale • B. Christophe · B. Foulon • P. Touboul • L. Maleki • N. Yu •

S. G. Turyshev • J. D. Anderson • F. Schmidt-Kaler • R. Walser • J. Vigué •

M. Büchner • M.-C. Angonin - P. Delva • P. Tourrenc $\cdot$ R. Bingham • B. Kent •

A. Wicht • L. J. Wang • K. Bongs · Hj. Dittus • C. Lämmerzahl • S. Theil •

K. Sengstock • A. Peters • T. Müller • M. Arndt • L. Iess • F. Bondu •

A. Brillet • E. Samain · M. L. Chiofalo · F. Levi • D. Calonico

Received: 13 March 2008 / Accepted: 18 September 2008 / Published online: 18 December 2008

(C) Springer Science + Business Media B.V. 2008

W. Ertmer · C. Schubert · T. Wendrich · M. Gilowski · M. Zaiser · T. v. Zoest · E. Rasel Institut für Quantenoptik, Leibniz Universität Hannover, Hannover, Germany

E. Rasel $(\varangle)$

Institut für Quantenoptik, Leibniz Universität Hannover, Welfengarten 1,

30167 Hannover, Germany

e-mail: rasel@iqo.uni-hannover.de

Ch. J. Bordé · A. Clairon · Landragin · P. Laurent · P. Lemonde · G. Santarelli

LNE-SYRTE, CNRS, Observatoire de Paris, Paris, France

W. Schleich · R. Walser

Institut für Quantenphysik, Universität Ulm, Ulm, Germany

M.-C. Angonin · P. Delva · P. Tourrenc

Université P. et M. Curie, ERGA, Paris, France

F. S. Cataliotti $\cdot$ M. Inguscio $\cdot$ N. Poli $\cdot$ F. Sorrentino $\cdot$ C. Modugno $\cdot$ G. M. Tino

LENS, Università di Firenze, INFN, Florence, Italy

P. Gill · H. Klein · H. Margolis

National Physical Laboratory, Teddington, UK

S. Reynaud · A. Lambrecht

LKB, ENS, CNRS, UPMC, Paris, France

C. Salomon

Ecole Normale Supérieure-Dép. de Physique, Paris, France 
Abstract In response to ESA's Call for proposals of 5 March 2007 of the COSMIC VISION 2015-2025 plan of the ESA science programme, we propose a M-class satellite mission to test of the Equivalence Principle in the quantum domain by investigating the extended free fall of matter waves instead of macroscopic bodies as in the case of GAUGE, MICROSCOPE or STEP. The satellite, called Matter Wave Explorer of Gravity, will carry an experiment to test gravity, namely the measurement of the equal rate of free fall with various isotopes of distinct atomic species with precision cold atom interferometry in the vicinity of the earth. This will allow for a first quantum test the Equivalence Principle with spin polarised particles and with pure fermionic and bosonic atomic ensembles. Due to the space conditions, the free fall of Rubidium and Potassium isotopes will be compared with a maximum accelerational sensitivity of $5 \cdot 10^{-16} \mathrm{~m} / \mathrm{s}^{2}$ corresponding to an accuracy of the test of the Equivalence Principle of 1 part in $10^{16}$. Besides the primary scientific goal, the quantum test of the Equivalence Principle, the mission can be extended to provide additional information about the gravitational field of the earth or for testing theories of fundamental processes of decoherence which are investigated by various theory groups in the context of quantum gravity phenomenology. In

E. Peik

Physikalisch Technische Bundesanstalt, Braunschweig, Germany

C. Jentsch · U. Johann · A. Rathke

EADS-Astrium, Friedrichshafen, Germany

P. Bouyer

Laboratoire Charles Fabry, CNRS, Institut d`Optique Théorique et Appliquée,

Palaiseau, France

L. Cacciapuoti

ESA, ESTEC, Noordwijk, The Netherlands

P. De Natale

INOA-CNR, Florence, Italy

B. Christophe $\cdot$ B. Foulon $\cdot$ P. Touboul

ONERA, Chatillon, France

L. Maleki $\cdot$ N. Yu $\cdot$ S. G. Turyshev

Jet Propulsion Laboratory, NASA, Pasadena, CA, USA

J. D. Anderson

Global Aerospace Corporation, El Segundo, CA, USA

F. Schmidt-Kaler

Quanten-Informationsverarbeitung, Universität Ulm, Ulm, Germany

J. Vigué · M. Büchner

LCAR, Université Paul Sabatier and CNRS, Toulouse, France

R. Bingham · B. Kent

Rutherford Appleton Laboratory, Didcot, UK 
this proposal we present in detail the mission objectives and the technical aspects of the proposed mission.

Keywords Test of equivalence principle - Fundamental physics • General relativity $\cdot$ Atom interferometer $\cdot \mathrm{MWXG}$

\section{Introduction}

A better understanding of gravity is today one of the most important issues in fundamental physics. Gravity is at the heart of some of the most fascinating and radical insights and findings in physics such

- as the recent revolutionary changes in cosmology which are motivated by observations e.g. made by WMAP. They recommend the existence of dark matter and dark energy which are only communicating with our world through gravity.

\footnotetext{
A. Wicht

Institut für Experimentalphysik, Heinrich-Heine-Universität Düsseldorf, Düsseldorf, Germany
}

L. J. Wang · K. Bongs

University of Birmingham, Birmingham, UK

Hj. Dittus · C. Lämmerzahl · S. Theil

ZARM, University of Bremen, Bremen, Germany

K. Sengstock

Institut für Laserphysik, Universität Hamburg, Hamburg, Germany

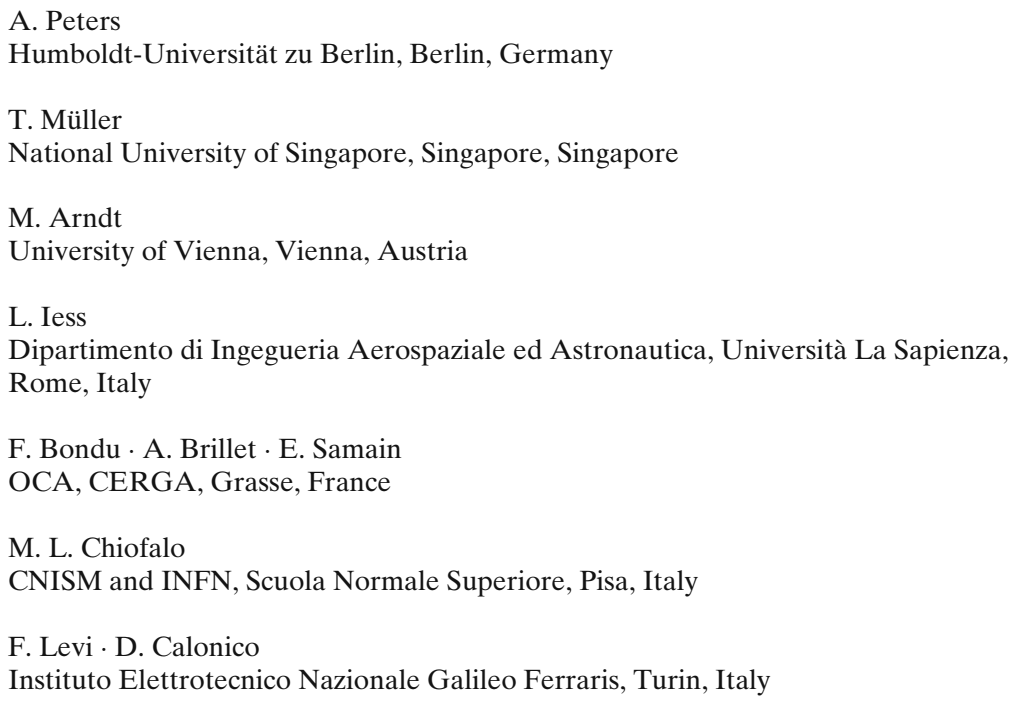

A. Peters

Humboldt-Universität zu Berlin, Berlin, Germany

T. Müller

National University of Singapore, Singapore, Singapore

M. Arndt

University of Vienna, Vienna, Austria

L. Iess

Dipartimento di Ingegueria Aerospaziale ed Astronautica, Università La Sapienza, Rome, Italy

F. Bondu · A. Brillet - E. Samain

OCA, CERGA, Grasse, France

M. L. Chiofalo

CNISM and INFN, Scuola Normale Superiore, Pisa, Italy

F. Levi · D. Calonico

Instituto Elettrotecnico Nazionale Galileo Ferraris, Turin, Italy 
- theories, which are expected to reconcile quantum mechanics and gravity. They predict new forces which will lead to a modification of the universal free fall.

- the puzzling findings from the Pioneer mission which eventually suggest an additional constant acceleration of unbound objects travelling through our solar system.

- theories predicting anomalous coupling between gravity and spinors, which represents a testground for general relativity and new theories beyond our standard models.

The principle of equivalence, which postulates that the gravitational mass equals the inertial mass, is intimately connected with gravity. Bodies of different mass and composition should display an equal rate of free fall. Tests of the fundamental postulate date back to early experiments by Galileo and are continuously improved by modern pendulum experiments and lunar laser ranging to an accuracy of 1 part in $10^{13}$. An improvement by several orders of magnitude is expected from test performed during the extended free fall of macroscopic bodies in earth-near orbits. The most important advantages space offers cold for cold atom interferometer are

- firstly, that in space, on a drag-free platform, gravitational perturbations can be reduced by 12 orders of magnitude. While there are other limitations and disturbances in space, the overall gain is still several orders of magnitude,

- and secondly, that space permits to extend the free fall in such a way that the required sensitivity can be achieved.

We propose to perform a test of principle of equivalence in the quantum domain. MWXG will investigate the free fall of different atomic species by atom-interferometric methods towards the earth. In this experiment, the atomic test particles have to be treated as matter waves, objects which are fully described by the Schrödinger wave equation according to the formalism derived by C. Bordé and coworkers. The wealth of atom-optical tools allows preparing ultra pure ensembles of matter waves of different spin polarization as well as pure bosonic and fermionic ensembles. Therefore, these methods venture into a new range of tests of the principle of equivalence. MWXG will operate with isotopes of rubidium and potassium. During about 2 years, MWXG aims to compare the rate of free fall of matter waves at a level of $5 \cdot 10^{-16} \mathrm{~m} / \mathrm{s}^{2}$.

\section{Scientific objectives}

2.1 The equivalence principle in the classical and quantum domain

The Equivalence Principle postulates the equivalence between inertial and gravitational mass, or stated differently, that bodies of different mass and/or 
composition fall with the same acceleration in a uniform gravitational field. It implies that all forms of energy (gravitational, nuclear, electro-magnetic, kinetic, thermal ...) contribute in an identical way to the heavy and inertial mass. This contention cannot be proven; it can only be tested to higher and higher precision.

Einstein generalised the Equivalence Principle and made it the foundation of his theory of General Relativity. A violation of the Equivalence Principle at some level would either require a modification of Einstein's theory or constitute the discovery of a new force. There are, in fact, good reasons to believe that General Relativity is not the ultimate theory of gravity. Gravitation, electromagnetism and the weak and strong interactions are the four known fundamental forces of Nature. Einstein's theory of gravity, General Relativity, provides the basis for our description of the Big Bang, the cosmological expansion, gravitational collapse, neutron stars, black holes and gravitational waves. It is a "classical", non-quantum field theory of curved spacetime, constituting an as-yet unchallenged description of gravitational interactions at macroscopic scales. The other three interactions are dealt with by a quantum field theory called the "Standard Model" of particle physics, which accurately describes physics at short distances where quantum effects play a crucial role. But, at present, no realistic theory of quantum gravity exists. This fact is the most fundamental motivation for pursuing our quest into the nature of gravity.

The Standard Model successfully accounts for all existing non-gravitational particle data. However, just as in the case of General Relativity, it is not a fully satisfactory theory. Its complicated structure lacks an underlying rationale. Even worse, it suffers from unresolved problems concerning the violation of the charge conjugation parity symmetry between matter and antimatter and the various unexplained mass scales. Purported solutions of these shortcomings typically involve new interactions that could manifest themselves as apparent violations of the Equivalence Principle.

The truly outstanding problem remains the construction of a consistent quantum theory of gravity, a necessary ingredient for a complete and unified description of all particle interactions. Superstring theories-in which elementary particles would no longer be point-like - are the only known candidates for such a grand construction. They systematically require the existence of spinless partners of the graviton: dilatons and axion-like particles. The dilaton, in particular, could remain almost massless and induce violations of the Equivalence Principle at a level that—albeit tiny—may well be within the reach of space missions.

A violation of the principle of equivalence leads to a modification of the gravitational law, for example by a new composition dependent inverse square law with charges $q_{1}$ and $q_{2}$.

$$
V=V_{\text {gray }}+V_{\text {unknown }}=-G \frac{m_{1} m_{2}}{r}-\frac{q_{1} q_{2}}{r}
$$

There are more general expressions for possible modifications o the gravitational attraction by additional interactions. The composition dependent 
modification can be parameterised as a function obeying the same or a different power law as well as by a Yukawa-type potential. A new composition dependent interaction can be described by dilaton scenarios or scalar-tensor theorie assigning the test particles a new charge which is related to a new form of coupling $\mathrm{V}_{\mathrm{NF}}$.

$$
V_{\mathrm{NF}}= \pm \frac{g_{\mathrm{NF}}}{4 \pi} q_{1} q_{2} \frac{e^{-r / \lambda}}{r}
$$

where $g_{\mathrm{NF}}$ is the coupling constant of the novel force, $\mathrm{q}$ denotes the attributed charge of a test particle, $\lambda=\hbar / m_{\mathrm{b}} \mathrm{c}$ is the Compton wavelength of the virtual exchange boson, and the minus and plus signs refer to interactions mediated by scalar or vector bosons, respectively. As the present experiments provide no bias what the "charge" of the Equivalence Principle violating interaction could be, one generally assumes the "charge" to be a function of the quantum numbers of its constituents. The most general vector charge of electrically neutral and stable matter is described as a function of the baryon number and the lepton number or as a function of the number of protons $(\mathrm{Z})$ and neutrons $(\mathrm{N})$. In order to choose elements as 'different' as possible, procedures have been established [1] allowing one to judge the sensitivity of material combinations for the detection of EP violations.

2.2 Classical tests: ground-based searches and limits, lunar-laser ranging (LLR)

There is a long history of testing the principle of equivalence with macroscopic bodies, which continuously improved the measurements. Historically, there have been four distinct methods of testing the Equivalence Principle:

- Galileo's free-fall method

- Netwon's pendulum method

- Netwon's celestial method (based on observations of the Earth-Moon system or of the moons of Jupiter in the Sun's field)

- Eötvös' torsion-balance method.

The most accurate tests have been the torsion-balance experiments and the celestial method. The milestones have been the pioneering experiment of Eötvös [2], its extension by Eötvös, Pekár and Fekete [3], the experiment by Roll, Krotkov and Dicke [4], the experiment by Braginsky and Panov [5], and a continuing series of state-of-the-art measurements by Adelberger et al. $[6,7]$. The current limits of torsion-balance and lunar-laser-ranging experiments are almost the same although they test slightly different things. The torsion-balance experiments have also been essential in the search for new composition dependent forces over scale lengths down to a few millimetres. The figure below is a summary of the results obtained by selected experiments over the last 100 years. 
The most precise tests today have achieved an accuracy of 1 part in $10^{13}$. They are based on modern torsion pendulum experiments, which probe the gravitational attraction of Titanium and Berylium to the earth, the sun and dark matter. A similar accuracy is achieved by Lunar Laser Ranging which compares the free fall of the earth and the moon towards the sun.

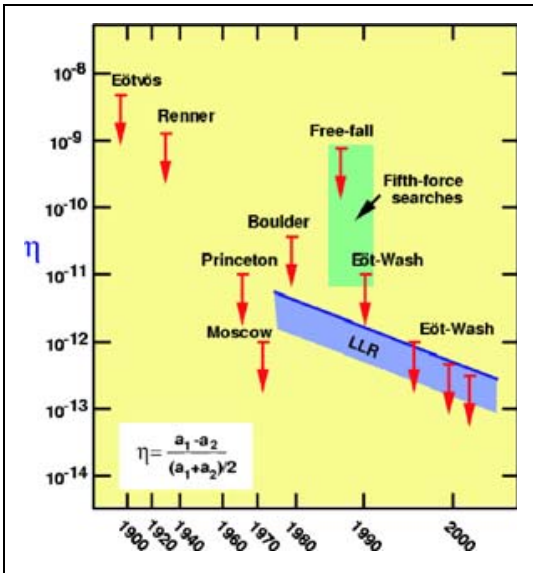

Figure: Selected tests of the weak equivalence principle as a function of $\eta$ , which measures fractional difference in acceleration of different materials or bodies. The free-fall and Eöt-Wash experiments were originally performed to search for a fifth force (green region, representing many experiments). The blue band shows evolving bounds on for gravitating bodies from lunar laser ranging (LLR).

Picture taken from [29]

\subsection{Classical tests with macroscopic bodies in space}

Experiments on the ground are limited because of the limited driving acceleration and the unavoidable and unshieldablemicroseismicity in Earth-based laboratories. Experiments on the ground are limited at this level because of unshieldable seismic noise and the weak driving acceleration. In space, this test could be done a factor $10^{3}$ to $10^{5}$ more precisely.

Probably the first test in space will be the CNES-led MICROSCOPE (MICRO-Satellite à TrainéeCompensée pour l'Observation du Principe d'Équivalence) project, a room-temperature test of the Weak Equivalence Principle to 1 part in $10^{15}$ [8]. The drag-compensated satellite will be in a Sun-synchronous polar orbit at $700 \mathrm{~km}$ altitude, with a payload consisting of two differential accelerometers, one with elements made of the same material (platinum rhodium alloy), and another with elements made of different materials (platinum rhodium alloy and titanium alloy). Differential displacements between the test masses of a pair are measured by capacitive sensors for over 1 year.

Another, known as Satellite Test of the Equivalence Principle (STEP), is under consideration as a possible joint effort of NASA and the European Space Agency (ESA), with the goal of a $10^{-17}$ test. STEP would improve upon MICROSCOPE by using cryogenic techniques to reduce thermal noise, among other effects. At present, STEP (along with a number of variants, called MiniSTEP and QuickSTEP) has not been approved by any agency beyond the level of basic design studies or supporting research and development. A variant of the STEP proposal is also proposed for Cosmic Vision: GrAnd Unification 
and Gravity Explorer (GAUGE). The cryogenic (few K) payload carries 3 classical test-mass pairs for a high-precision EP test: one test-mass pair for macroscopic $1 / \mathrm{r}^{2}$ and spin coupling tests, and a spin-coupling source mass. Depending on the feasibility, GAUGE will also carry an atom interferometer on board to investigate the free fall of matter waves and decoherence effects of matter waves.

\subsection{Quantum tests of the equivalence principle}

Quantum tests of the equivalence principle investigate the propagation of matter waves in a gravitational field. Gravity acts on matter waves similar like a material with an inhomogeneous index of refraction on light waves, which bends the straight trajectories of light waves to parabolas. According to the Schrödinger equation the bending should not depend on the nature of the matter waves. The centre of mass of packets of matter waves are expected to follow the trajectory of classical bodies, while they disperse in width. In this respect, classical mechanics can be seen as the limit of geometrical optics of quantum waves.

The phase induced by accelerations is measured by atom-interferometric methods. Atomic interferometers represent a novel technology of quantum devices for ultra-precise sensing and monitoring of accelerations and rotations. The potential of atom interferometers and atom lasers can be compared with Superconducting Quantum Interference Device (SQUID) sensors, but without need for cryogenic equipment. Atomic inertial quantum sensors function similar like atomic clocks, which are today the most-accurate standards for time and frequency measurement. Like atomic clocks, which revolutionised frequency metrology, inertial and rotational sensors using atom interferometers display a high potential for replacing classic state-of-the-art sensors. The recent improvement of the sensor performance is mainly due to the rapid progress in the laser development and in the manipulation of atoms.

MWXG will apply atomic Mach-Zehnder-type interferometers. They are predestined for measurements of gravity due to its high symmetry. In a MachZehnder interferometer atomic wave packets made out of cold atoms are coherently split, re-directed and re-combined to observe matter wave interferences. Beam splitting is achieved by the atom-light interaction. During each interaction sequence the atoms are exposed to a pulsed light field generated by two counter-propagating laser beams. An atom absorbs a photon out of one laser beam and is stimulated by the other laser beam to re-emit the photon. In this way twice the recoil of a photon is transferred coherently to the atomic wave (rather than atoms) to generate a new spatial mode of a matter wave. For a Mach-Zehnder-type atom interferometer the phase shift due to accelerations a can be calculated by the following formula

$$
\Delta \Phi_{\mathrm{acc}}=\vec{k}_{\mathrm{eff}} \cdot \vec{a} T^{2}=\vec{k}_{\mathrm{eff}} \cdot \vec{a} \frac{L^{2}}{v^{2}}
$$



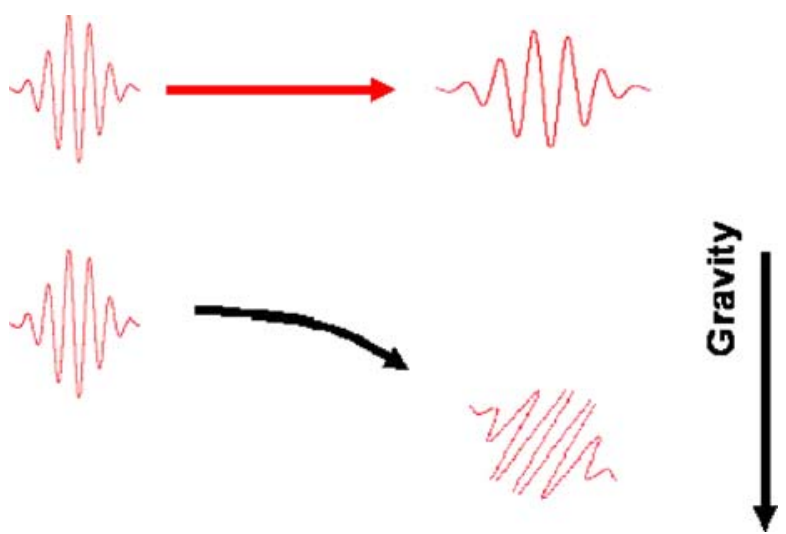

Freely falling wave packets without and with gravity

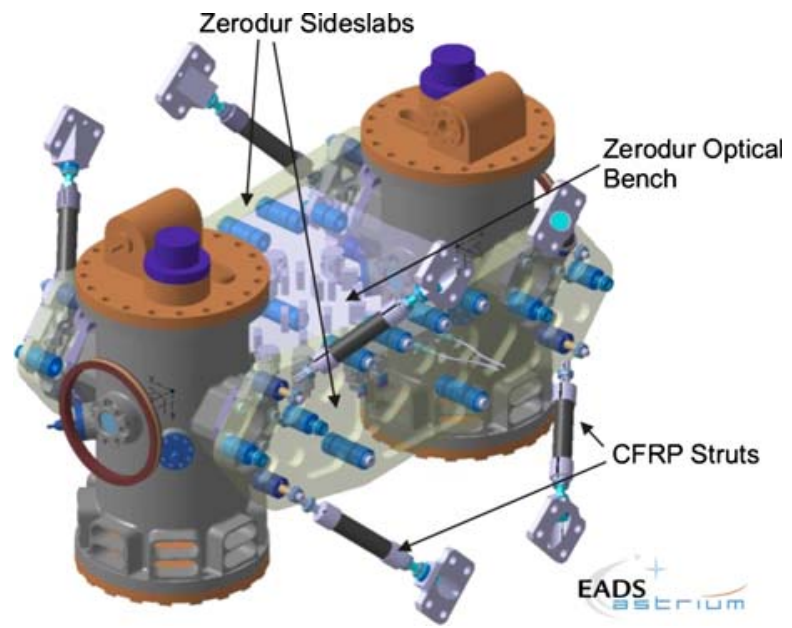

Picture: Astrium GmbH - Satellites, Friedrichshafen

The equation is derived from the Schrödinger equation and describes the gravitational phase shift of matter waves in a Mach-Zehnder type atom interferometer. The equation has important implications: The phase shift depends only on the acceleration, the momentum difference of the two interfering matter waves and the square of the drift time of the matter waves inside the interferometer. This simplicity recommends atom interferometer as inertial references for measuring absolute accelerations. The momentum difference of the two interfering spatial matter wave modes is determined by the photon recoil of the light beam interacting with the matter wave, a well defined quantity. The equation states also the equality of the inertial and the gravitational mass as she is independent of the atomic mass of the particular species. 
The sensitivity of the atomic accelerometer increases for a given length scale $L$ of the detection volume and effective photon recoil $k_{\text {eff }}$ of the interferometer with the square of the atomic drift time $\mathrm{T}$ or the inverse square of the atomic velocity. Thus, for an atomic accelerometer the sensitivity as well as the precision by using ultra-cold atoms in a space-bound experiment would increase by several orders $\left(10^{3}\right.$ to $\left.10^{4}\right)$ of magnitude allowing longer measurement times) and providing a more stable environment. According to the equation the Mach-Zehnder interferometer senses accelerations in only one particular direction defined by the coherently scattered photons.

The Mach-Zehnder interferometer displays analogies to the drag-free sensors based on the optical read out of the position of a macroscopic proof-mass with a light interferometer as employed by LISA [9] and LPF. In principle, one replaces the macroscopic proof mass by an ensemble of cold atoms. Form this point of view, the atom interferometer employs the atoms as test masses and senses accelerations with respect to the payload/satellite frame.

MXWG will search for deviations of the free propagation of matter waves of different isotopes of different atomic species in the terrestrial gravitational field. The techniques of laser cooling allow for preparing pure ensembles with a specific spin state and specific quantum statistics, i.e. bosonic and fermionic ensembles of atoms. On the other hand, the applicability of these techniques restricts the list of appropriate candidates. Candidate species for which laser cooling has been successfully demonstrated and their properties are given in the following tables

\begin{tabular}{ll}
\hline Table on candidate species & \\
\hline Rubidium & ${ }^{85 / 87} \mathrm{Rb}$ \\
Caesium & ${ }^{133} \mathrm{Cs}$ \\
Potassium & ${ }^{40 / 41} \mathrm{~K}$ \\
Lithium & $6 / 7 \mathrm{Li}$ \\
Ytterbium & ${ }^{171-176} \mathrm{Yb}$ \\
Strontium & ${ }^{87} \mathrm{Sr}$ \\
\hline
\end{tabular}

\begin{tabular}{lll}
\hline Table listing the features of $\mathrm{Rb}, \mathrm{K}$ & & \\
\hline Quantity & ${ }^{85} \mathrm{Rb}$ & ${ }^{41} \mathrm{~K}$ \\
\hline $\mathrm{B}$ & 85 & 41 \\
$\mathrm{~L}$ & 37 & 19 \\
$\mu_{\mathrm{S}} / \mu$ & -0.0123 & -0.011 \\
$\mu_{\mathrm{EM}} / \mu$ & $2.71 \cdot 10^{-3}$ & $1.8 \cdot 10^{-3}$ \\
$\mu_{\mathrm{W}} / \mu$ & $6.8 \cdot 10^{-7}$ & $2.4 \cdot 10^{-7}$ \\
\hline
\end{tabular}

Here $B$ and $L$ are the baryon and lepton number. $\mu_{\mathrm{S}} / \mu, \mu_{\mathrm{EM}} / \mu$ and $\mu_{\mathrm{W}} / \mu$ are the fractional mass of the test bodies due to strong, electromagnetic and weak interaction, respectively.

Favourable candidate are the isotopes Rubidium and Potassium because they behave very similar regarding their chemical, optical and atom-optical features. 


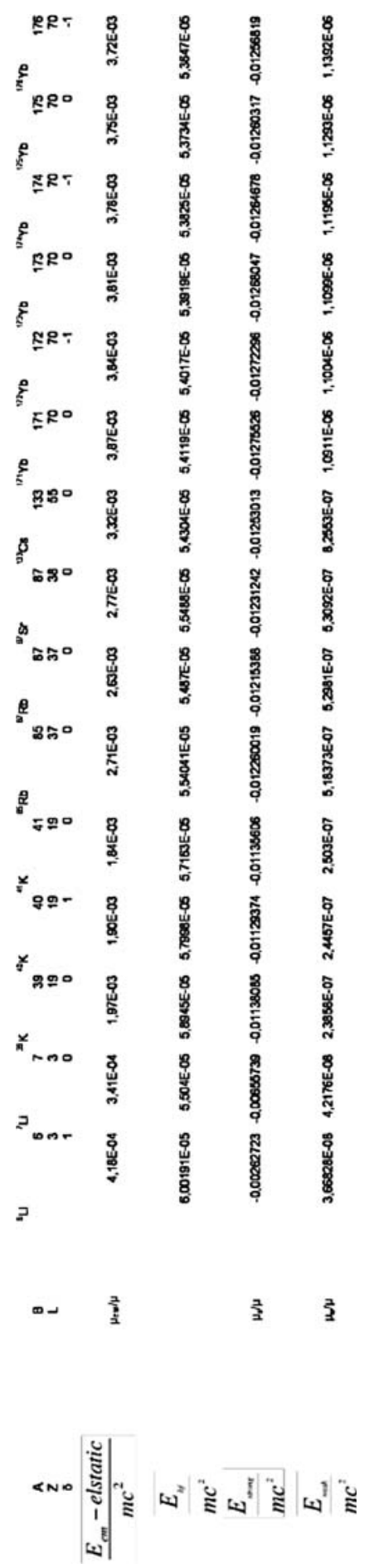


For comparing the free fall of matter waves of rubidium and potassium, MWXG will synchronously operate two atom interferometers. This means that preparation of the matter wave packets by laser cooling and trapping as well as their coherent manipulation during the interferometer cycle, has to be done at the same time. For signal separation, the detection of the interferometer output after the interferometer cycle will be done successively in a narrow time window of few milliseconds. The synchronous operation guarantees a suppression of common mode accelerations with respect to the satellite, which was demonstrated on ground-based experiments up to $140 \mathrm{~dB}$. The use of $\mathrm{K}$ and $\mathrm{Rb}$ facilitates the synchronous operation and automaticaly guarantees alignment of the two interferometers as the laser light for manipulation for cooling, detection and coherent manipulation can be delivered in one fibre.

MXWG will make pair wise comparisons between different isotopes of rubidium and potassium while it orbits around the earth. For one comparison, the targeted sensitivity will enable to reach the level sensitivity, i.e. $5 \cdot 10^{-16} \mathrm{~m} / \mathrm{s}^{2}$ for an integration time of about 3 months. Variation of crucial parameters (magnetic field, atomic temperature, polarisation and density of the atomic ensembles), in between the actual measurements, will extend the mission to about 2 years.

The signal induced by a possible modification of the Equivalence Principle will oscillate while the satellites orbits around the earth as the atom interferometer senses the acceleration in only one direction and the satellites orientation changes with respect to the earth as shown in the following figure. The signal period corresponds to an orbital day.

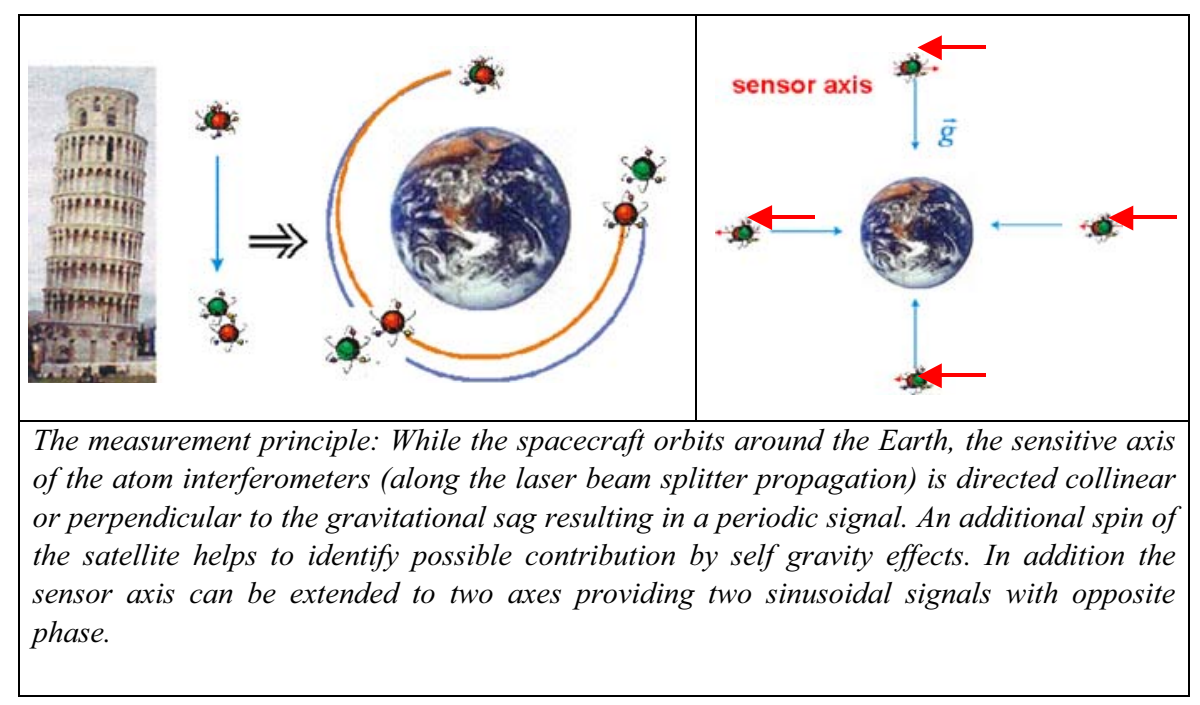

The redundancy of the payload could be enhanced by adding a second pair of atom interferometers. If the two pairs of atom interferometers are oriented in orthogonal direction, two complementary signals are generated with $90^{\circ}$ phase shift. The upgrade would mainly effects the weight of the satellite, as 
the two interferometers can be operated such that the same laser can be used for both interferometers. Slowly spinning the satellite (with less than the earth rotation rate) would provide additional information about the self-gravity of the satellite and systematic effects.

\subsection{Drag-free sensor: comparing microscopic and macroscopic test bodies?}

The atom interferometers sample the acceleration with about a frequency of $0.3 \mathrm{~Hz}$. The period of the measurement cycle is determined by the preparation of the atoms $(<1 \mathrm{~s})$, the interferometry cycle $(2 \mathrm{~s})$ and the detection $(<0.1 \mathrm{~s})$. Due to the dead time, the rms residual accelerations of the satellite have to be below $10^{-9} \mathrm{~m} / \mathrm{s}^{2}$, such that signal of the interferometer remains at the central fringe. In order to achieve the targeted sensitivity, MWXG requires a threeaxis stabilised, drag-free spacecraft. For the attitude control the satellite has to carry a drag-free sensor such as the

- STAR \& SUPERSTAR, fabricated by ONERA, based on capacitive readout, or

- the LPF-type sensor based on optical read out of the position of a macroscopic proof mass.

In contrast to the proven technology of STAR and SUPERSTAR with capacitive read out, the optical read-out will be demonstrated for the first time by the Pathfinder mission. The use of the STAR or SUPERSTAR sensors is fully compatible with the requirements of $\mathrm{MWXG}$ and has the advantage of less weight and power consumption. The LPF-type sensor, however, might be of interest for comparisons of this technique with atomic sensors, as they operate in a similar frequency band. In addition, the laser for atom interferometry could be used at the same time for the optical read out of the LPF-type sensor. The coupling to the drag-free proof masses is reduced with respect to capacitive read-out.

The comparison of the classical drag-free sensors with the atomic quantum sensor can be interpreted as test of the equivalence principle of macroscopic bodies with microscopic bodies, namely the atomic species. As both sensors can hardly be superposed, the achievable accuracy depends on the precise modeling of the self-gravity of the spacecraft and the precise knowledge of the centre-of-mass position of the macroscopic and microscopic test masses.

The comparison of both techniques for inertial sensors is also of interest as inertial sensors are at the heart of missions such as GRACE or GOCE as well as a key-technology for LISA and forth-coming generations of gravitational wave detectors.

A pair of inertial sensors provides a gravi-gradiometer can be used for geodetic purposes and provide complementary data to ongoing satellite mission by measuring the cross-track gradient of the spatial variations of the Earth's gravitational field along the orbit [10]. However, the chosen orbital altitude of at least 1,000 km (a required for compensated the atmospheric drag at the required level) will reduce the spatial resolution and accuracy. 
2.6 Current status of matter-wave interferometry and prospects

of ground-based studies

The potential of matter wave sensors have been demonstrated by laboratory prototypes more than 10 years ago. A high sensitivity of atom interferometers has been demonstrated by the measurement of $g$, the local acceleration due to gravity, with a precision of about $10^{-9}$ by the group of S. Chu [11]. An other very promising variant of atomic interferometer was the gravity gradiometer developed by the group of M. Kasevich. This sensor measures the gradient of gravity $\Delta \mathrm{g}$ with a precision approaching state-of-the-art mobile gravitygradient sensors [12].This experiment is very similar to the gravity meter of S. Chu using two atomic interferometers which are roughly displaced by $1 \mathrm{~m}$. However, instead of using two independent atomic interferometers the remarkable precision and robustness of this configuration is mainly achieved by using one Raman laser system for both interferometers at the same time.

Considering the atomic interferometer of $\mathrm{S}$. Chu, a comparison with a Michelson gravimeter based on falling corner cubes, (FG-5 produced by Micro-g-Solutions, Arvada, Colorado) with a quoted relative uncertainty of $2 \mathrm{ppb}$ showed a difference of $7 \pm 7 \mathrm{ppb}$ due to the uncertainty of the gravity gradient measurement of $5 \mathrm{ppb}$. The resolution of the atom interferometer has been about four times higher than the FG-5 due to the higher repetition rate, the noise behaviour was similar. Limitation were the measurement time and, thus, the large gravitational acceleration.

Since these pioneering experiments, these sensors have been further developed for special applications and transportable sensors. Most prominent examples are the atomic sensors developed by M. Kasevich at Stanford university or the gravi-gradiometer at the JPL by Nan Yu in the US as well as current developments at the SYRTE (Paris), the LENS (Florence), Leibniz Universität Hannover and Humboldt Universität zu Berlin. These activities comprise sensors to measure the Newtonian constant, terrestrial gravity and the Earth rotation rate.

\section{Mission profile proposed to achieve these objectives}

\subsection{Orbit requirements}

A circular orbit will enable residual geodesic motion of the test particles on a nearly equi-potential surface due to the limited change of the gravity gradient. In addition, a Sun-synchronous dawn-dusk orbit is preferred where the fixed sun pointing will allow minimising temperature fluctuations and thus providing a thermally stable environment. In addition, eclipse time is relatively short and there is only one eclipse season.

The orbit altitude is a trade-off between the scientific requirements for a sufficiently low orbit to detect the gravitational force as well as to minimise the radiation exposure, and the need to minimise atmospheric drag and eclipse 
duration. At an altitude of $1,000 \mathrm{~km}$, the atmospheric air density is between 2.5 $\times 10^{-15}$ and $1.7 \times 10^{-13} \mathrm{~kg} / \mathrm{m}^{3}$ depending on the solar activity. This translates into a force between $1.49 \times 10^{-7}$ and $1.01 \times 10^{-5} \mathrm{~N}$ per m${ }^{2}$ of cross-sectional area.

After comparisons of the major orbit perturbations like residual drag, radiation pressure from Sun or Earth albedo or gravity gradients, an altitude between 700 and $1,000 \mathrm{~km}$ is reasonable. However, $1,000 \mathrm{~km}$ has been chosen in order to minimise the atmospheric drag and therefore enable optimisation of the drag-free performance. Finally, we arrive at the following mission profile:

\begin{tabular}{ll}
\hline Orbit parameter & Parameters chosen for MWXG \\
S/c & 3-axis stabilized \\
Type & Circular, sun-synchronous, dawn-dusk \\
Altitude & $1,000 \mathrm{~km}$ \\
Period & $105.12 \mathrm{~min}$ \\
Inclination & $99.48^{\circ}$ \\
Maximum eclipse duration & $822 \mathrm{~s}(13.7 \mathrm{~min})$ \\
Length of eclipse period & 57 days \\
Nominal lifetime & 2 years \\
\hline
\end{tabular}

\subsection{Launcher requirements}

The most cost effective launcher for MWXG is VEGA. The launcher performance is sufficient to lift the MWXG satellite with a total mass of $723.6 \mathrm{~kg}$ directly to a sun-synchronous circular orbit at $1,000 \mathrm{~km}$ altitude with an inclination of $99.48^{\circ}$. There will even be enough mass margin for the case that in a mission definition study a higher altitude will be chosen. The launch site will be Kourou. The moderate cost for a VEGA launch amounts about $22 \mathrm{M} €$. In addition Vega's injection accuracy of typically $5 \mathrm{~km}$ in altitude, $0.05^{\circ}$ in inclination and $0.1^{\circ}$ in RAAN is sufficient to arrive at the required parameters. The Rockot launch vehicle also shows sufficient performance for the MWXG satellite launch mass for the given orbit parameters and can therefore be considered as back-up solution. Both Vega and Rockot fairings provide enough space to integrate the MWXG spacecraft.

\begin{tabular}{ll}
\hline Table: Launcher requirements & \\
\hline Launcher & VEGA \\
Launch mass & $723.6 \mathrm{~kg}$ \\
Launch site & Kourou \\
Launch cost & $22 \mathrm{M} €$ \\
Launcher & VEGA \\
Launch mass & $723.6 \mathrm{~kg}$ \\
\hline
\end{tabular}

\subsection{Mission lifetime}

The mission lifetime is mainly determined by the limited thrusters' lifetime for the drag-free control (FEEPs are baselined). Appropriate FEEP performance 
will be demonstrated in earlier missions like LPF and Microscope scheduled for the timeframe 2009-2012. It is therefore not considered to be critical. If nevertheless it turns out that the thrusters' behaviour differs from the requirements there exist promising alternatives like cold gas thrusters or colloid thrusters. A period of 2 years will be sufficient to perform several successive calibration and science measurement campaigns to average the measurements down to the envisaged level defined in the science goals.

\subsection{Ground segment requirements}

The Launch and Early Orbit Phase (LEOP) will be supported by three stations from the ESA LEOP ground station network (Kiruna, Kourou, Perth). Injection will be in visibility from Kourou. Due to the launcher dispersion, the injection orbit may not be exactly Sun-synchronous. This may lead to a RAAN shift of not more than $1.5^{\circ}$ after 1 year. This is perfectly acceptable. Therefore no orbit maintenance other than the drag-free system is needed. The most suitable ESA ground station for an SSO is Kiruna. Characteristics of the coverage are summarised in the Table below:

\begin{tabular}{ll}
\hline Table: Characteristics of MWXG ground coverage from Kiruna \\
\hline Minimum elevation & $5.0 \mathrm{deg}$ \\
Mean pass duration & $13.3 \mathrm{~min}$ \\
Mean number of passes per day & 10.5 \\
Ground contact per day & $140 \mathrm{~min} /$ day \\
\hline
\end{tabular}

\subsection{Critical issues}

The MWXG mission requirements are driven by the science objectives. The most stringent of those requirements, which essentially drive the spacecraft system design, are the following:

- means of compensating accelerations to $10^{-10} \mathrm{~g}$ at $0.3 \mathrm{~Hz}$ along the sensors sensitive axis

- mission lifetime 2 years

- Technology Readiness Level (TRL) 4-5 (i.e. "Component/breadboard tested in relevant environment").

The de-orbiting of the spacecraft at end of life (EOL) is not imposed, but it is considered desirable.

\subsection{Overview of all proposed payload elements}

The payload consists of

- the atom interferometers,

- a rigid optical bench and

- a drag-free proof mass. 
The atom interferometers comprises several subsystems

- Atom manipulation unit, with the atomic sources and the fibre access for the laser to manipulate the atoms

- Laser bench hosting the laser generating the light for cooling, trapping, detecting and coherent manipulation of the atoms

- Ultra-low noise microwave sources serving as reference for the atom interferometer

The payload will have similarities with LPF regarding the required functional elements such as the need for an optical bench, a more complex (with respect to LPF) laser bench and a vacuum chamber for the atom manipulation (atoms are replacing the drag-free proof mass). The LPF payload design is shown in the figure below.

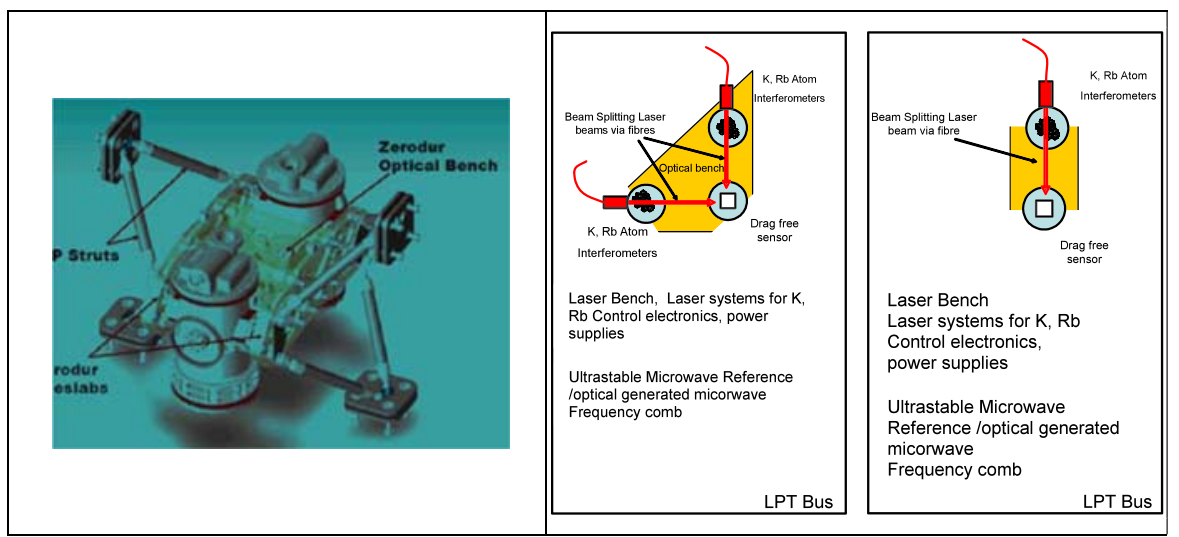

Figure: on the left side, a drawing of the LPF payload; on the right side a schematic of the payload of MWXG, comprising the configuration for 2 pairs of double atom interferometers and a drag free proof mass and the configuration for 1 double atom interferometer.

\subsection{Summary of each instruments key resource characteristics}

\subsubsection{Atom interferometer}

The core element of the payload is a pair of dual atom interferometers. The pair is measuring accelerations in two orthogonal directions with respect to the proof mass. Each of the two units consists of a dual-species atom interferometer $(\mathrm{Rb} / \mathrm{K})$ to perform the differential acceleration measurement between to atomic species of different mass.

For achieving an optimal suppression of common-mode-noise, which accords to basically all possible disturbance sources, the two atomic species are simultaneously prepared, coherently manipulated and detected with optimally overlapped atomic clouds of the two species.

Only one dual atom interferometer is required to perform the test of the equivalence principle. Mass and power can be reduced by this configuration. 
Appropriate atomic species as candidates for high precision tests of the EP by means of atom interferometer accelerometers are ${ }^{85 / 87} \mathrm{Rb}$ and ${ }^{40 / 41} \mathrm{~K}$. With the choice of these atoms the payload will be optimised from the technical point of view as it will be possible to run the experiments with the same laser sources. In addition investigations like comparing the free fall of bosons and fermions offer to extend the science output. The most important atomic data is given in table below.

The rate of fall of potassium and rubidium will be measured independently with two atom interferometers. A precise comparison of the two results is only possible if the two measurements are performed at the same time and at the same environmental conditions. Therefore, both atomic ensembles will be captured together first in a magneto-optical trap. This technique allows confining simultaneously $10^{10}$ atoms of each species at the same place at the $\mu \mathrm{m}$ level. Both atomic ensembles can be prepared with similar, rather small velocities of less than $1 \mathrm{~cm} / \mathrm{s}$ such that they expand equally. The small velocities as required for the measurement are achieved by laser cooling and adiabatic expansion. A more precise positioning can be achieved with a dipole trap. However, trapping of more than $10^{9}$ in a dipole trap has to be demonstrated.

The signal of the two atomic species can be discriminated by their fluorescence which differs sufficiently in wavelength.

The limitations of this test are set by effects which affects distinctively the two atomic species. The experimental configuration rules out several such problems. The centres of mass of both atomic clouds coincide due to the symmetric expansion, even though the velocities of both atomic species might slightly differ. However, as the two species differ considerably in mass and the momentum transferred by the beam splitter is nearly equal, the separation of the wave packets is about two times larger for potassium. This can be partially compensated by transferring the twice or a fraction of the effective recoil on the rubidium atoms. Consequently, any perturbation, like gravity gradients or residual accelerations or rotations on the spacecraft should affect both atomic species equally and cancel. An excellent common-mode rejection can be also achieved for other noise sources, such as the phase noise caused by the beam splitting (using the same quartz oscillator for the two microwave synthesizers needed for the beam-splitting lasers), the noise due to aliasing (since both atomic species interact at exactly the same time with their respective beamsplitting laser) and the vibratory and rotational noise (by using the same optical elements for both beam-splitting lasers). The ultimate limitations are possibly due to interactions between the atoms themselves and the distinct light shifts sensed by the atoms when they interact with the beam-splitting lasers. The light shifts, however, can be possibly measured using in addition a microwave clock.

For performing the different steps required in the Atom interferometer the following subsystems are utilized:

Atom preparation bench The Atom Preparation Bench rigidly holds two magnetically shielded vacuum chambers, which constitute the atom interferometers. On the vacuum chambers, the atomic reservoirs and the optics for the 


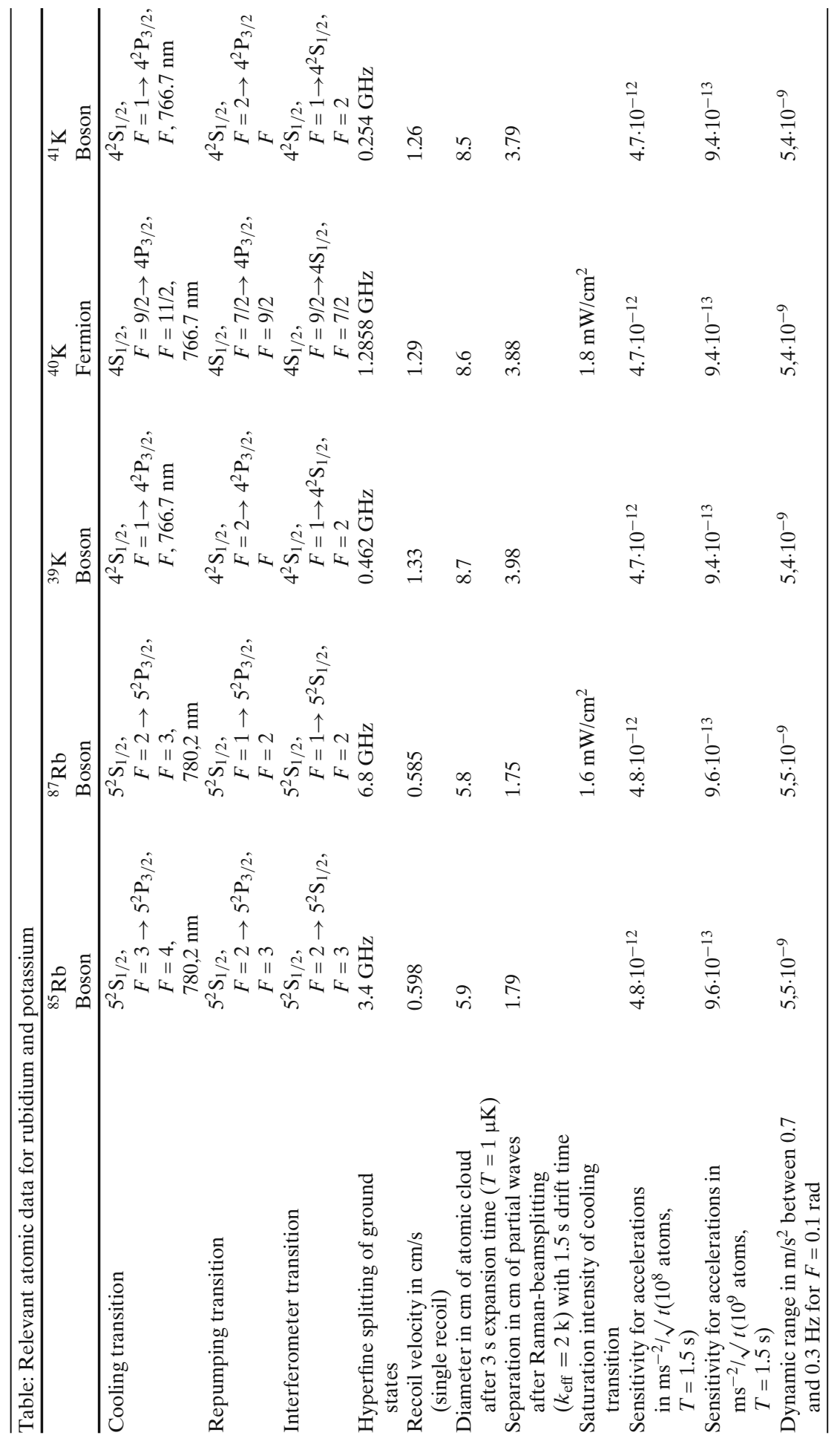


preparation and detection of the atoms are mounted. The Atom Preparation Bench is made of a carbon-composite structure holding the Optical Bench in its centre. Together with the optical bench, it constitutes the Payload Module (PLM).

Atom interferometer optics The light for coherent splitting and recombination of the atomic waves is delivered from the Laser bench to the Atom Preparation Bench using optical fibers, which guarantees stable beam alignment at the fiber exits. For assuring a precisely controlled and stable alignment of the atom interferometer light beam-splitters, the fiber output couplers and further optics as for example mirrors are mounted on the Rigid Optical Bench, which is described in a following section.

Laser bench The Laser Bench is a panel of the spacecraft primary structure which houses less sensitive, mainly optical components for the atom interferometers (lasers, power supplies, control electronics). The light is guided via optical fibres to both the Atom Preparation Bench and the Optical bench. For both atomic species, high-power, maintanence free diode-laser systems are utilized, which have already been developed in a similar setup for the PHARAO project. As the laser wavelengths used for the manipulation of the two species differ only slightly ( $780 \mathrm{~nm}$ for $\mathrm{Rb}$ and $767 \mathrm{~nm}$ for $\mathrm{K}$ ), the same optical elements can be used.

Ultra low-noise microwave source An ultra low-noise radio-frequency oscillator is used in the Atom interferometer for different purposes as all relevant parameters of the AIF operation are in some way referenced to a frequency, which can be controlled extremely precise. The most stringent requirements are placed by the phase-stabilization of the Raman-lasers, which has to be kept at a level of minimum phase noise, as Raman-laser phase-noise will appear as accelerational noise in the interferometer and concerning a non-perfect common-mode noise suppression.

Rigid optical bench The Optical Bench consists of an ultra-stable monolithic Zerodur structure with an integrated drag-free sensor and the optical elements (mirrors and fibre optic couplers) which control the beam splitting and recombination in the atom interferometers. With this arrangement all the critical elements are rigidly mounted with very high thermo-mechanical stability. The Optical Bench is located in the centre of the spacecraft and is mounted on the Atom Preparation Bench. Insulation and isostatic mounting provide thermal and structural de-coupling.

\subsubsection{Drag-free proof masses and drag-free control}

During science operations, a drag-free control system uses the drag-free sensors to drive the spacecraft FEEP thrusters, in order to maintain a near 
zero-gravity environment for the payload. The basic principle of drag-free control is to use a "free-floating" mass, shielded from external disturbances, as a test mass within the spacecraft. The displacement between this mass and the spacecraft is then measured very accurately. As the spacecraft is subject to external disturbances, this then acts as a measure of those disturbances. By using these measurements to control the thrust applied to the spacecraft, the spacecraft will "chase after" the "disturbance-free" mass. This method of control leads to very small residual accelerations on the spacecraft. A dragfree sensor is being developed under the TRP program for use on LPF and possibly on LISA and GOCE, which will be used in a similar configuration in MWXG.

Drag-free control (and hence the very low accelerations) can only be effected at one point (nominally the centre of the test mass for a single-sensor system). The further from this test mass, the larger the residual accelerations become. These accelerations come mainly from three effects: gravity gradient due to the extended nature of the spacecraft, spacecraft self gravity, and angular rotations due to instabilities in the pointing. Other contributions, from sensor noise and third body (lunar) interactions, are expected to be second-order effects. The acceleration requirements for MWXG apply to the entire interferometer volume. It is therefore obvious to minimise the distances between the proof mass and the atom interferometers. In order to balance the spacecraft self gravity, optional correction masses around the test mass housing will be introduced in the design. These test masses can than be integrated during the assembly phase according to the resulting self gravity imbalance as simulated.

\subsection{Performance assessment with respect to science objectives}

Testing the EP to a level of $5 \cdot 10^{-16} \mathrm{~m} / \mathrm{s}^{2}$ implies

- The measurement of accelerations with the AIF to the same level of precison

- A noise level of accelerations provided by the drag-free control not limiting the accelerational measurement in the desired frequency range

\subsubsection{Atom interferometer}

The measurement principle of the atom interferometer is based on the determination of the phase shift $\varphi$ induced by accelerations $\mathbf{a}$ in the direction of the momentum $\mathbf{k}$ transferred by the beams-splitters onto the atoms

$$
\varphi=\vec{a} \vec{k} T^{2},
$$

where $T$ is the evolution time between the interferometer beam-splitters. Following this formula, one can assess the sensitivity to accelerations by the 
resolution of the interferometers phase shift $\varphi$ and the scaling factor $\boldsymbol{k} T^{2}$ of the interferometer. The resolution of the interferometers phase shift is ultimately limited by shot-noise of the splitted atomic waves, which is given by $1 / \mathrm{N}^{1 / 2}$, with $\mathrm{N}$ the number of atoms contributing to the interferometer signal. With 2 . $10^{9}$ detected atoms, a drift time of $1 \mathrm{~s}$, and a transferred momentum given by the wavelength $\lambda$ of the 2 -photon Raman-transition with $\lambda=2 \pi / \mathrm{k}$ we expect a accelerational sensitivity of $1.24 * 10^{-12} \mathrm{~m} / \mathrm{s}^{2} \mathrm{~Hz}^{-1 / 2}$ for $\mathrm{Rb}$ and $1.22 * 10^{-12} \mathrm{~m} / \mathrm{s}^{2}$ $\mathrm{Hz}^{-1 / 2}$ for $\mathrm{K}$. Integration of the measured accelerations during 100 days will therefore lead to an accelerational sensitivity of $0.72 \cdot 10^{-15} \mathrm{~m} / \mathrm{s}^{2}$ for $\mathrm{Rb}$ and $0.71 \cdot 10^{-15} \mathrm{~m} / \mathrm{s}^{2}$ for $\mathrm{K}$, including a typical preparation time of $1 \mathrm{~s}$ before each interferometer measurement. This corresponds to a test of the principle of equivalence of one part in $10^{16}$. A more conservative boundary condition is a $\mathrm{S} / \mathrm{N}$ ratio of 10.000 corresponding to a test with an statistical accuracy of 5 parts in $10^{16}$. The condition can be even more relaxed when different data sets of the 2-year measurement can be combined. In this case the requirements on the atomic sources are drastically relaxed as only $10^{8}$ detectable atoms have to be generated. The resolution can be further enhanced by increasing the effective recoil transferred by the atom-light beam splitting process. However, the transfer of more than 4 photon recoils includes a large translation of the atomic wave packets (compare ch. 2 and 4.2) along the laser beam, which imposes additional constraints to the modelisation of gradient and the overlap of the trajectories. The increase to 4 photon recoils would result in a shortening of the integration time by a factor 4 , i.e. less than 1 month or reduce the requirement on the atomic source, i.e. trapping of $2 \cdot 10^{7}$ atoms.

\subsubsection{Laser}

Phase/frequency control requirements Both the relative phase noise between the two laser fields used for the Raman pulses and their absolute frequency noise may limit the sensitivity of acceleration measurement. With suitable choice of experimental configuration most noise sources can be made common mode in the differential acceleration measurements, and the correlated limiting effects can be reduced by orders of magnitude. Anyway, the laser phase/frequency stability may still be critical to the target sensitivity of $6 \cdot 10^{-16}$.

Relative phase noise of Raman laser fields The transfer function that maps relative phase noise of Raman laser fields onto atomic accelerometer sensitivity has been derived and experimentally verified in [arXiv:physics/0510197, 2006]. The results agree with an intuitive qualitative model: since the interferometric sequence consists of three Raman pulses of length $t$ separated by a time $T$, the interferometer should filter out the noise above $f_{h} \approx t^{-1}$ and below $f_{l} \approx T^{-1}$. For a target resolution $d f$ in the interferometer phase, it is necessary that the integrated relative phase noise of the Raman laser fields between $f_{l}$ and $f_{h}$ be lower than $d f$. 
The relative frequency between the two Raman laser fields is set by an ultrastable oscillator at the ground-state hyperfine splitting frequency $(6.8 \mathrm{GHz}$ for $87 \mathrm{Rb}, 1.3 \mathrm{GHz}$ for $40 \mathrm{~K}$ ). Assuming for such oscillator a white phase noise spectrum in the region [ $f ; f h]$, with $t=10 \mu$ s the target sensitivity $d f \approx 0.1 \mathrm{mrad}$ requires a phase noise level around $-130 \mathrm{dBc} / \mathrm{Hz}$, which is not extremely challenging. However, close to $f_{l}$ the phase noise density usually rises as $1 / f$ due to unavoidable flicker noise. In [Appl. Phys. B 84, 673 (2006)] it has been shown that the low frequency term can be dominant, especially for the case of high sensitivity (large $T$ ) measurements. Using the same reference oscillator to synthesize the $1.2 \mathrm{GHz}$ and the $6.8 \mathrm{GHz}$ signals allows noise rejection to some extent as common mode, if the two synthesis chains transpose the performance of the reference source without serious degradation. Quantitatively, the flicker noise of the $100 \mathrm{MHz}$ reference oscillator developed for the PHARAO space clock would limit the resolution of an interferometer with $T=5 \mathrm{~s}$ to more than $10 \mathrm{mrad}$. Assuming to lock both the $1.3 \mathrm{GHz}$ and $6.8 \mathrm{GHz}$ oscillators to a $100 \mathrm{MHz}$ reference with the same flicker noise performance, the target resolution of $d f \approx 0.1 \mathrm{mrad}$ requires a common-mode noise rejection of at least $20 \mathrm{~dB}$. Such level of noise rejection has been already demonstrated, for instance in [Appl. Phys. B 84, 673 (2006)].

The stable synthesized microwave frequency (either $6.8 \mathrm{GHz}$ or $1.3 \mathrm{GHz}$ ) must be phase-coherently transferred to the relative frequency between the pair of Raman laser fields. This can be done either by direct sideband generation into an electro-optic or acousto-optic modulator, or by phase-locking two independent laser sources. Though the latter solution seems to be more convenient in terms of power consumption, it would more likely result in excess phase noise added by the optical PLL. So far, the best performance of optical phase-locked loops has never resulted in a phase noise level better than $-120 \mathrm{dBc} / \mathrm{Hz}$ below $100 \mathrm{kHz}$, see for instance [Appl. Phys B 84, 633 (2006)].

An important advantage of measurement in microgravity is that the requirements on phase linearity of the synthesized frequency are much released. In ground atom interferometers the atoms undergo a Doppler shift of several $\mathrm{MHz}$ due to free-fall acceleration, and the frequency difference of the two Raman laser fields must be changed correspondingly to keep in resonance with the falling atoms. Controlling the microwave electronics along a large frequency sweep is not trivial at the level of precision required for a resolution of $d f \approx 0.1 \mathrm{mrad}$, due to possible nonlinear frequency dependent phase shifts (in RF filters, amplifiers, electro-optic or acousto-optic modulators).

Absolute frequency noise of Raman laser fields The interferometer phase also depends on the absolute frequency of the Raman laser fields, due to several independent effects. While the differential light shift can be eliminated with suitable choice of the intensity ratio between the Raman laser fields, still the delay induced by the difference of the laser beams paths makes the interferometer sensitive to the fluctuations of the frequency of the lasers [arXiv:physics/0701023v1, 2007]. In principle, it is possible to minimize such effect by reducing the relative time delay, that is, by making the path length 
difference close to zero. However, this would not be the most convenient geometry, since the phase difference is also affected by fluctuations of the respective paths of the two Raman laser beams over the propagation distance. The influence of path length variations is minimized by overlapping the two beams, and making them propagate as long as possible over the same path. However, since the two Raman fields must interact with the atoms with opposite wave-vectors, it is necessary to retro-reflect the overlapped beams after they pass through the atoms. As a consequence, the reflected beam is delayed with respect to the other one.

If the absolute frequency of the Raman laser fields drifts on a time scale short compared to the interrogation time $T$ between pulses, this can cause an asymmetric phase shift to be read into the atomic coherence due to this path asymmetry. The corresponding excess phase noise $\delta \phi \approx 2 \pi t_{d} \delta v$ is proportional to the time delay $t_{d}=2 L / c$ induced by the beam path difference $2 L$ and to the integrated frequency noise $d n$ of the Raman beams over the Fourier frequency range $[f ; f h]$ defined by the interferometer period $T$ and pulse duration $t$. For a white laser frequency noise $S_{f}$ of the Raman laser beams, the corresponding phase uncertainty will be:

$$
\delta \phi \approx \frac{\pi t_{d}}{2} \sqrt{\frac{S_{v}}{\tau}}
$$

where $S_{v}$ is the power spectral density of laser frequency noise. Assuming a time delay of $2 \mathrm{~ns}(L=30 \mathrm{~cm})$ and $t=10 \mu \mathrm{s}$, a target resolution of $0.1 \mathrm{mrad}$ gives a limit on laser frequency noise $S_{v}<10^{4} \mathrm{~Hz}^{2} / \mathrm{Hz}$, corresponding to a fast laser linewidth lower than $32 \mathrm{kHz}$. Such noise level is not straightforward with diode lasers, but it may be at hand with some care on the laser frequency stabilization. However flicker frequency noise may easily become dominant, especially with large $\mathrm{T}$, and its control may be rather challenging. It will be convenient to relax the demand on laser frequency stability by reducing the mirror distance $L$ and increasing the pulse duration $t$.

\subsubsection{Drag free control}

A drag-free sensor (used here essentially as an accurate accelerometer) is being developed under the TRP program for use on LPF and possibly on LISA and GOCE. The specification placed on this development is for a measurement accuracy of $1 \times 10^{-14} \mathrm{~m} / \mathrm{s}^{2} / \sqrt{ } \mathrm{Hz}$ in the frequency range 0.001 to $0.1 \mathrm{~Hz}$. It is expected that this allows sufficient performance for MWXG, even when operated at the higher frequency of $0.6 \mathrm{~Hz}$ versus $0.1 \mathrm{~Hz}$ and used in a different operational mode ("strap-down" mode as opposed to "free-flying" mode). The noise on the development sensor is specified as $1 \times 10^{-10} \mathrm{~m} / \sqrt{ } \mathrm{Hz}$, for MWXG this translates to a noise level of approximately $2 \times 10^{-11} \mathrm{~g}$. The performance of the sensor, once developed, will need to be proven in flight on LPF. 
The drag-free performance of the Pathfinder platform as optimised for operation at L1 is determined by a combination of the residual free fall of the test mass to $\Delta \mathrm{a}_{\mathrm{FF}}<3 \cdot 10-14 \mathrm{~m} / \mathrm{s}^{2} / \sqrt{ } \mathrm{Hz}$ and the distance jitter between the spacecraft and the test mass $\Delta x_{S / C, T M}$. The residual acceleration noise can be calculated with the relation $\Delta a_{S C} \approx\left(\Delta \mathrm{a}_{\mathrm{FF}}^{2}+\left(\Delta^{2} \Delta x_{S / C, T M}\right)^{2}\right)^{1 / 2}$ depending on the Fourier frequency wu For the LPF parameters of $\Delta x_{S / C, T M}<$ $5 \mathrm{~nm} / \sqrt{ } \mathrm{Hz}$ at $1 \mathrm{mHz}$ this results in $\Delta a_{S C}<2 \cdot 10^{-13} \mathrm{~ms}^{-2} / \sqrt{ } \mathrm{Hz}$. However, as the payload will be placed in a distance 1 of typically $40 \mathrm{~cm}$ from the drag-free point, it occurs an additional contribution due to the angular acceleration (typically $8 \cdot 10^{-12} \mathrm{~s}^{-2} / \sqrt{ } \mathrm{Hz}$ around $1 \mathrm{mHz}$ ) multiplied by the lever arm length 1 . In total, we have a residual acceleration of $\Delta a_{S C \text {,total }} \approx$ $\left(\left(2 \cdot 10^{-13} \mathrm{~ms}^{-2} / \sqrt{ } \mathrm{Hz}\right)^{2}+\left(8 \cdot 10^{-12} \mathrm{~s}^{-2} / \sqrt{ } \mathrm{Hz} \times 1 / 1 \mathrm{~m}\right)^{2}\right)^{1 / 2}$. In total, for $l=0.4 \mathrm{~m}$ we result in $\Delta a_{S C \text {,total }} \approx 3.2 \cdot 10^{-12} \mathrm{~m} / \mathrm{s}^{2} / \sqrt{ } \mathrm{Hz}$. This performance will be reached at $1 \mathrm{mHz}$ and slightly degrade for $>0.1 \mathrm{~Hz}$. However, the calculation of the dynamic range shows that the required residual accelerations must be kept smaller than $4 \cdot 10^{-9} \mathrm{~m} / \mathrm{s}^{2} / \sqrt{ } \mathrm{Hz}$. Therefore, in principle a drag-free proof mass system like in LTP is not required from the performance point of view but for the direct optical readout.

\subsection{Resources: mass, volume, power, OBDH and telemetry}

All budgets are assumption relying on LPF, Hyper and PHARAO data. Especially in the Pharao cold atom clock experiment similar payload units have been developed and can thus be taken as authentic reference.

\section{1) Atom interferometer budgets}

This budget contains already two experimental chambers for simultaneously probing $\mathrm{K}$ and $\mathrm{Rb}$. In addition, part of the lasers installed on the cooling and detection laser package are will be used for the optical readout of the drag-free test mass.

\begin{tabular}{lcccc}
\hline & Mass $(\mathrm{kg})$ & $\begin{array}{l}\text { Mass incl } \\
\text { margin }(\mathrm{kg})\end{array}$ & Power $(\mathrm{W})$ & $\begin{array}{l}\text { Power incl } \\
\text { margin }(\mathrm{W})\end{array}$ \\
\hline Physics package & 45.1 & 54.1 & 23.0 & 27.6 \\
$\begin{array}{l}\text { Cooling and detection } \\
\quad \text { laser system }\end{array}$ & 32.0 & 38.4 & 52.0 & 62.4 \\
$\begin{array}{l}\text { Raman laser system } \\
\text { Femtosecond comb }\end{array}$ & 24.0 & 28.8 & 40.0 & 48.0 \\
$\quad \begin{array}{l}\text { reference } \\
\text { Optical fibers }\end{array}$ & 7.6 & 9.1 & 24.0 & 28.8 \\
$\begin{array}{l}\text { Harness } \\
\text { Computer control }\end{array}$ & 1.6 & 1.9 & 0.0 & 0.0 \\
$\quad$ and interface & 11.0 & 2.4 & 0.0 & 0.0 \\
Total & 123.3 & 13.2 & 22.2 & 26.6 \\
\hline
\end{tabular}

The central PLM will house the 2 atom interferometer devices, the dragfree sensor as well as the connecting optical bench. All other units will 
be accommodated in outer compartments of the LPF structure. A budget breakdown is shown below:

\begin{tabular}{lcccc}
\hline & Mass $(\mathrm{kg})$ & $\begin{array}{l}\text { Mass incl. } \\
\text { margin }(\mathrm{kg})\end{array}$ & Power $(\mathrm{W})$ & $\begin{array}{c}\text { Power incl. } \\
\text { margin }(\mathrm{W})\end{array}$ \\
\hline $\begin{array}{l}\text { Payload module } \\
\text { Physics package }\end{array}$ & 45.1 & 54.1 & 23.0 & 27.6 \\
$\begin{array}{l}\text { Optical bench } \\
\text { Drag-free sensor }\end{array}$ & 15.8 & 17.7 & 0.0 & 0.0 \\
Total & 56.0 & 63.0 & 20.0 & 23.0 \\
Other payload subsystem units & 116.9 & 134.8 & 43.0 & 50.6 \\
$\begin{array}{l}\text { Cooling and detection } \\
\quad \text { laser }\end{array}$ & 32.0 & & & \\
$\begin{array}{l}\text { Raman laser } \\
\text { Femtosecond comb }\end{array}$ & 24.0 & 38.4 & 52.0 & 62.4 \\
$\quad$ reference & 7.6 & 28.8 & 40.0 & 48.0 \\
$\begin{array}{l}\text { Optical fibers } \\
\text { Harness }\end{array}$ & 1.6 & 9.1 & 24.0 & 28.8 \\
$\begin{array}{l}\text { Comp. control and } \\
\text { interface }\end{array}$ & 2.0 & 1.9 & 0.0 & 0.0 \\
dfs electronics & 11.0 & 13.2 & 0.0 & 0.0 \\
Total & 40.0 & 45.0 & 22.2 & 26.6 \\
\hline
\end{tabular}

\section{2) Drag-free test mass}

This budget comprises one single test mass as well as the required electronics and the optical bench for connecting the AIs with the DFS. The optical readout has already been included in the AI budget above.

\begin{tabular}{lcccc}
\hline & Mass $(\mathrm{kg})$ & $\begin{array}{l}\text { Mass incl } \\
\text { margin }(\mathrm{kg})\end{array}$ & Power $(\mathrm{W})$ & $\begin{array}{c}\text { Power incl } \\
\text { margin }(\mathrm{W})\end{array}$ \\
\hline $\begin{array}{l}\text { Optical bench } \\
\begin{array}{l}\text { Drag-free sensor } \\
\text { test mass }\end{array}\end{array}$ & 15.8 & 17.7 & 0 & 0 \\
dfs electronics & 56.0 & 63.0 & 20 & 23 \\
Total & 40.0 & 45.0 & 16 & 18.4 \\
\hline
\end{tabular}

OBDH and telemetry The science data will be handled and analysed by the payload computer which controls the different science modes of the experiment such as

- Measurement-mode, as well as the initialisation modes such as

- test modes for the atom interferometers,

- bias field measurement and adjustment,

- demagnetisation,

- calibration,

- laser source initialisation.

The payload computer will be responsible for the proper timing of the experimental sequences, for the choice of parameter and sampling and monitoring. Depending on the sampling rate $(1-10 \mathrm{~s})$ the two atom interferometers 
will deliver output signals, which will be converted to accelerations. These signals will be evaluated under consideration of the signal of the drag-free sensors. The key parameters of the monitoring of the experiment will be preassessed, selected and, if significant, for the interpretation of the experiment stored and transmitted.

The overall science data rate is expected to be similar to LPF, a rough estimate expects to below $0.2 \mathrm{kbps}$ or roughly $1.2 \mathrm{Mb}$ per orbital period.

\subsection{Pointing and alignment requirements}

The pointing and alignment requirements of the optics correspond to LPF.

\subsection{Calibration requirements and operating modes}

- Magnetic field shifts

- Spacecraft selfgravity

- Temperature, density dependence of signal

- Light shift

- Senstitivity to temperature variations

In the normal operation mode a typical atom interferometer cycle of slightly more than $3 \mathrm{~s}$ duration will be run. It consists of the following steps:

- $100 \mathrm{~ms}$ of charging and laser cooling the atoms where the atoms are trapped from residual background pressure and laser-cooled down to residual temperatures of the order of 1 micro Kelvin.

- $50 \mathrm{~ms}$ ground state preparation where the atoms are pumped into the magnetic insensitive ground state by optical and/or microwave means. The residual atoms in unwanted states are blown away by a blaster beam.

- $30 \mathrm{~ms}$ of switching off the trapping light fields and applying the right currents for the homogeneous interferometry magnetic field. About $30 \mathrm{~ms}$ are required in order to damp out effects due to the current switching.

- $3 \mathrm{~s}$ of interferometer cycle consisting of 3 Raman pulses of about $10 \mathrm{~ms}$ duration separated by $1.5 \mathrm{~s}$

- $50 \mathrm{~ms}$ of detection where the populations of the two ground states are detected by a calibrated photodiode system.

A specific calibration for the performance assessment of the atomic sensors will be required in order to investigate all possible error sources as well as the accuracy of such a device. In addition to these technical tests the interferometer itself can be interchanged such that the acceleration sensitivity nearly vanishes (characterisation of all other non-acceleration noise sources), and the sensitive direction can be reversed by either reversing the Raman laser beams or inverse the ground state after preparation. Another important requirement will be to vary the time $\mathrm{T}$ between the Raman laser pulses in order to build interferometers with moderate acceleration sensitivities when reducing $\mathrm{T}$. This will also allow characterising the fringe pattern and setting the AI to its working point. During science run the most critical parameters like 
the laser beam powers have to be monitored online and will be recalibrated in the order of once per day.

\subsection{Special requirements (including, if appropriate, proposed ESA provided items)}

All atom interferometer packages will carry at least three layers of Mumetal shielding against external magnetic fields. In addition, several magnetic coils in Helmholtz or anti-Helmholtz configuration will be used to generate the required fields for the interferometry as well as actively compensating external fields.

\subsubsection{Control of the selfgravity of the satellite}

- Mass distribution and Gravity gradients

- Satellite rotation

\subsubsection{Residual accelerations and rotation rates}

The critical frequency range is defined by

- the sampling rate of the atom interferometer $(0.3 \mathrm{~Hz}$ as well as multiple frequencies) due to aliasing effects

- the dynamic range of the atom interferometer $(0.3 \mathrm{~Hz}$ to $0.01 \mathrm{~Hz})$ and

- the characteristic frequency of the signal, i.e. the orbital frequency + space craft role.

The atom interferometer AIF does not continuously monitor the accelerations. The sampling rate is determined by the duration of a full measurement cycle (incl. trapping, preparation, interferometry and detection of the atoms) and the sensitivity selected for the AFI. The sampling time is $3 \mathrm{~s}$. Due to aliasing the AIF transforms frequencies in the vicinity to the sampling frequency (and multiple frequencies) to lower frequencies. Therefore perturbations with Fourier frequencies close by the sampling frequency are critical.

A continuous change in the acceleration is detected by the AIF as a linear phaseshift and, thus, transformed into a sinusoidal signal at the output of the AFI. The period of the oscillation depends on the sensitivity selected for the AFI. The AIF can give a well-defined information only if the displacement of the phase remains in the bounds of $\pm \pi$. This requirement defines the dynamic range of the AFI. The level of residual accelerations has to be such that the AFI sweeps not over a full period during the sampling (i.e. $3 \mathrm{~s}$ ).

Acceleration noise should not go beyond the admissible dynamic range of the atomic gyroscope. In the range of $0.3 \mathrm{~Hz}$ and $0.03 \mathrm{~Hz}$ the maximum amplitudes should consequently not exceed at least $10^{-10} \mathrm{~g} / \sqrt{ } \mathrm{Hz}$ for the lowfrequency AFI. Residual quasi-static accelerations in the frequency range of the orbital period $(<0.03 \mathrm{~Hz})$ should not exceed a level of $10-8 \mathrm{~g}$ at $0.003 \mathrm{~Hz}$. 
Magnetic fields Magnetic fields will be used for the magneto-optical trap, for the maintenance of the polarisation of the atoms, and for demagnetisation of the magnetic shields.

The coils are used in an anti-Helmholtz configuration, i.e. reversed electrical currents, which provides a quadrupole field with a field minimum and a linear field gradient at half the distance between the coils. The field gradient required by the magneto-optical trap is about $10 \mathrm{Gauss} / \mathrm{cm}$ ( $\mathrm{Rb}$ as well as $\mathrm{K})$. The atom interferometer is based on polarised atoms or, in other terms, on particular Zeeman quantum states which have no magnetic momentum and, thus, are less susceptible for magnetic fields. A bias field, which lifts the degeneracy of the Zeeman quantum states, is required to avoid depolarisation of the atoms. Four wires are tightened along the trajectories of the atoms such that they generate a homogeneous field of a strength of about $100 \mathrm{mG}$ with an orientation in direction of the beam-splitter laser. The homogeneity of the bias field is guaranteed by a stable mechanical mount. Temporal variations which could deform the mount of the coil are strongly reduced by the design and the materials selected for the payload module. Additional coils at the outside of the magnetic shields are required for demagnetisation of the magnetic shields at the initialisation of the experiment. They will consequently be used only temporally.

Magnetic shielding A double magnetic shield will reduce by a factor 10000 external stray fields, such as the fields caused by the Earth, solar activities or the satellites electricity, in the direction of the bias field. Magnetic fields due to the satellites electricity are further reduced by using twisted cables. The magnetic shielding of the complete Atom Preparation Bench is required as the atoms are in second order sensitive to magnetic fields. The projected sensitivity of the atom interferometers, however, is such that phase shifts due to magnetic fields of the second order have to be considered. The frequency shift between the two quantum states is about $5 \cdot 10^{-4} \mathrm{~Hz} / \mathrm{mG}^{2}$.

A constant homogenous field should not induce a phase shift at the interferometer output. After the first beam splitter, the atoms, which initially are all in the same quantum state, are equally split. One half of the atom remains unaffected, while the other half is deflected and transferred to the second quantum state. At the second beam splitter the atoms in both arms of the interferometer are re-directed to merge and interchange their quantum states. In each arm the atoms consequently spend half their time in one of the quantum states and thus the phase shift due to a homogenous field is cancelled. Homogenous fields and temporal variations of their field strength are uncritical for the AFI. The phase shift is equal for both counter-propagating atom interferometers and thus cancels out when the signals are subtracted.

The suppression of stray fields causing field gradients is more crucial because they break the symmetry between the two interferometers. However, they are only critical if they appear at the same Fourier frequency as the EP violating signal and if they point in direction of the bias field $(100 \mathrm{mG})$. A stray 
field of one $\mathrm{mG}$ lead to a frequency shift between the two quantum states of $86 \mathrm{mHz}$.

The influence of magnetic field can be further reduced by more sophisticated strategies of sampling. Unlike phase shifts due to rotations and accelerations, phase shifts due to magnetic field gradients only depend of the internal atomic states. Therefore, reversing the direction of propagation of the two Raman lasers only changes the sense of inertial phase shifts and, thus, can be discriminated form effects due to magnetic fields. In a similar manner, the role of the two quantum states, can be interchanged. In both cases, averaging over consecutive measurements (with opposite Raman directions or input states), will compensate the effect of magnetic field gradient. For field fluctuations the compensation is presumably not perfect but reduces the sensitivity to the magnetic gradient by the ratio of the period of oscillation to the time between the two consecutive measurements. The worst case, fluctuations at the Fourier frequency of the signal, have to be reduced to a level of $4 \mathrm{nG}$ over the full length of the interferometer. This requirement is achievable and should not limit the MWXG mission.

\subsection{Current heritage and technology readiness level (TRL)}

The PLM architecture and a preliminary design for the Optical Bench and Atom Preparation Bench were defined during the HYPER study. A Laser Bench concept is already existing (from the PHARAO experiment), while the basic atom interferometer components are existing as breadboard models (also from PHARAO). An optical design for the interferometer was provided by RAL, and its mechanical stability requirements are judged similar to the LISA requirements. Even though no drag-free control system is currently in existence, it is considered that the upcoming LPF drag-free control system can be adapted to the MWXG needs. Thermal stability, mechanical decoupling and stability were preliminarily assessed during the study. However, the overall system performance of MWXG should be further demonstrated by detailed design and analysis (i.e. drag-free control, pointing and thermo-elastic stability requirements) in a future Definition Phase. The atom interferometer level can be considered as TL 4-5.

\subsection{Critical requirements}

The sensitivity rises proportional to the square of the drift time. However due to the limited temperature of the atom cloud which leads to an expansion in diameter of $\approx 3 \mathrm{~cm} / \mathrm{s}$ the available payload volume limits drastically the residual free fall time and thus the drift time. In order to further improve these limits extensive work is ongoing looking into alternative atom trap and cooling schemes. For example optical trapping fields could be used to further confine the atoms or alternative cooling schemes could further reduce the temperature and therefore reduce the residual cloud expansion. However, these techniques would significantly increase the payload complexity and require additional 
mass and power resources and have therefore been disregarded for this baseline

\section{Basic spacecraft key factors}

\subsection{Number of spacecraft}

For the MWXG payload a single one drag-free spacecraft is required. Mass and power budgets as well as the most important parameters are given in the following sections. The LPF (LPF) bus has been chosen as baseline for the satellite platform with only small modifications. This will lead to a cost effective approach but also offers the opportunity to access already tested and approved hardware.

\subsection{Attitude and orbit control required: spinner/scanner/3-axis stabilized and associated requirements}

The MWXG is three-axis stabilised and during the science phase operates in a drag-free mode. Attitude is determined using a gyro-stellar estimator with inputs from 2 star trackers and inertial measurement units; in drag-free mode the LPF-sensor provides attitude information. Attitude control uses onboard FEEP micro-propulsion, study of the disturbance environment in the selected orbit will determine if further actuators are required and the amount of propellant. For some measurement phases the spacecraft needs to be spun along its long axis.

\subsection{On-board data handling and telemetry requirements}

The MWXG on-board data handling (OBDH) system is characterised by the continuous acquisition of low data rate from a single payload instrument that includes dedicated sensors to support the drag-free Secondary AOCS. The $\mathrm{OBDH}$ is also in charge of storing the science data during ground station nonvisibility for later downlink.

The On-Board Computer Unit (OBC) is internally redundant, including a Mass Memory Unit (MMU) of 2 Gbit, the interface to a redundant platform serial bus (typical CAN, OBDH or MIL-STD-1553) and I/Os modules as necessary to interface with the AOCS. The OBC is a complete redundant data management and control unit that provides telemetry and telecommand support functions, timing function, on-board surveillance and reconfiguration functions, and communications with PLM and SVM units through bus standard interfaces or dedicated interfaces (internal modular architecture).

The communications subsystem has to provide download for a data rate of $2.2 \mathrm{kbps}$ generated onboard ( $2 \mathrm{kbps}$ housekeeping, plus $0.2 \mathrm{kbps}$ science data or a total of $13.9 \mathrm{Mbits} /$ orbit). Any antenna aspect angle must be covered during LEOP and emergencies, while during operations antenna coverage 
must be compatible with the flight attitude and Sun-pointing of the spacecraft. The communication subsystem baseline design includes:

- S-Band Near Earth Transponder, with 2 W RF power coherent operation and ranging capability, $8 \mathrm{~W}$ Tx- and $5 \mathrm{~W}$ Rx-DC power consumption.

- Three antennas to maximise coverage: one wire antenna on $+X$, inserted in the solar array, and two helix semi-hemispherical antennas on -X, installed over booms.

- The resulting telemetry and telecommand rates are:

- 2 kbps TC with margins $>40 \mathrm{~dB}$

- $20 \mathrm{kbps}$ TM data rate for LEOP and emergency (NRZ/BPSK/PM modulation)

- $500 \mathrm{kbps}$ TM data rate for operations (BPSK or SPL/PM modulations).

The downlink approach envisages onboard storage of 13.9 Mbit per orbit (105 min) and of 190 Mbit per day (13.7 orbits). With a downlink at $500 \mathrm{kbps}$, 1-day data can be downloaded in a single pass (about $7 \mathrm{~min}$ ). For download every sixth day five passes are required within 1 day. Therefore, a 2 Gbit mass memory will be required to store the data acquired between successive downlink sessions.

\subsection{Mission operations concept (ground segment)}

All facilities established for MWXG will be based on extension of existing ground segment infrastructure, tailored to meet the requirement of the MWXG mission. The ground segment for MWXG in the operational configuration will consist of:

- The Ground Stations and the Communications Network

- The Mission Operations Centre (MOC), including infrastructure, computer hardware, the Flight Control System, data processing and flight dynamics software

- The Science Operations Centre (SOC), which is not part of the ESA ground segment but under science community responsibility.

Ground stations and communications network:

Due to the $98.2^{\circ}$ inclination, $1,000 \mathrm{~km}$ altitude Sun-synchronous orbit, it is assumed that the ESA S-band Station at Kiruna (15 $\mathrm{m}$ antenna) will be used for contact with the spacecraft during all mission phases. The Station will be used by the spacecraft at the maximum during one pass per day (alternatively: five passes every sixth day) for science data downlink and TT\&C with coverage intervals complementary to those of other missions.

During the first 15 days of the mission (LEOP) and during all critical missions phases, the ESA $15 \mathrm{~m}$ stations at Kourou and Perth will also be used. All ESA stations will interface to the MOC at ESOC in Darmstadt via the OPSNET communications network. 
The telemetry data rate from the spacecraft to the ground stations will be 500 kbps.

Mission operations centre (MOC) The MWXG mission will be operated by ESOC and will be controlled from the Mission Operations Centre (MOC) which basically consists of the Main Control Room (MCR), augmented by the Flight Dynamics Room (FDR), Dedicated Control Rooms (DCRs) and Project Support Rooms (PSRs). During periods around major mission events, mainly during launch and critical period of the transfer phase (LEOP and principal manoeuvre phases), the MCR will be used for MWXG mission control. During the science operations phase, the mission control will be conducted from a Dedicated Control Room.

The control centre is equipped with workstations giving access to the different computer systems used for different tasks of operational data processing. It will be staffed by dedicated MWXG operations staff and experts in spacecraft control. The computer configuration used in the MOC for the MWXG mission will be derived from existing infrastructure. The MOC will interface with the SOC (Science Operations Centre, under Science Community responsibility) via dedicated redundant lines. A Flight Control System based on infrastructure development (SCOS 2000), using a distributed hardware and software architecture for all spacecraft monitoring and control activities, will be established. Within the SCOS 2000 system, mission-specific software will be developed wherever necessary.

\subsection{Estimated overall resources (mass and power)}

The total spacecraft wet mass of $723.6 \mathrm{~kg}$ provides enough margin with respect to the VEGA launch performance to $1,000 \mathrm{~km}$ altitude $S S O$ of above $1,200 \mathrm{~kg}$ (Rockot performance is about $1,100 \mathrm{~kg}$ ). However, the power budget is marginal.. With a solar array area of $2.8 \mathrm{~m}^{2}$ of multi-junction GaAs an EOL power of $690 \mathrm{~W}$ should be feasible. In a more detailed study the power budget should be further investigated. An alternative would also be to alternate between the two atom interferometers. During the eclipse season we assume to reduce the required power by setting the atom interferometers to stand-by mode. This will sufficiently reduce the battery discharge during shadow and allow for efficient battery charging in Sun light.

\subsection{Specific environmental constraints (EMC, temperature, cleanliness)}

The general design strategy adopted is to de-couple the sensitive payload components as much as possible from the external disturbances. Gravitational, thermal and magnetic environmental constraints already in place for the overall LPF spacecraft will be well representative of those needed for MWXG. They will however need to be reassessed in relation to the specific mass distribution on the MWXG. Cleanliness constraints apply mainly to the drag-free sensor and the atom preparation unit. The most demanding EMC 


\begin{tabular}{|c|c|c|c|c|}
\hline & Mass (kg) & $\begin{array}{l}\text { Mass incl } \\
\text { margin }(\mathrm{kg})\end{array}$ & Power $(\mathrm{W})$ & $\begin{array}{l}\text { Power incl } \\
\text { margin }(\mathrm{W})\end{array}$ \\
\hline Payload & 235.1 & 273.7 & 197.2 & 234.8 \\
\hline Structure & 80.8 & 80.8 & 0.0 & 0.0 \\
\hline Thermal & 13.5 & 15.5 & 45.0 & 49.5 \\
\hline Solar array & 14.5 & 16.0 & 0.0 & 0.0 \\
\hline Power & 42.9 & 48.0 & 10.0 & 10.5 \\
\hline Propulsion & 41.1 & 47.1 & 220.5 & 221.2 \\
\hline AOCS & 14.2 & 14.3 & 32.8 & 33.5 \\
\hline OBDH & 15.0 & 17.3 & 38.0 & 39.9 \\
\hline TT/TC & 7.5 & 7.9 & 18.0 & 18.9 \\
\hline $\begin{array}{l}\text { Launch } \\
\text { vehicle } \\
\text { adapter }\end{array}$ & 60.0 & 60.0 & 0.0 & 0.0 \\
\hline $\begin{array}{l}\text { Gravitational } \\
\text { balance mass }\end{array}$ & 20.0 & 20.0 & 0.0 & 0.0 \\
\hline \multirow[t]{6}{*}{ Mass } & $\begin{array}{l}\text { Total satellite } \\
\text { dry mass }\end{array}$ & & $544.6 \mathrm{~kg}$ & \\
\hline & $\begin{array}{l}\text { Total satellite } \\
\text { dry mass incl. } \\
\text { maturity margin }\end{array}$ & & $600.5 \mathrm{~kg}$ & \\
\hline & System margin & & $120.1 \mathrm{~kg}$ & $20 \%$ \\
\hline & Total dry mass & & $720.6 \mathrm{~kg}$ & \\
\hline & Propellant cold gas & & $3.0 \mathrm{~kg}$ & \\
\hline & Total launch mass & & $723.6 \mathrm{~kg}$ & \\
\hline \multirow[t]{7}{*}{ Power } & Total power & & $561.5 \mathrm{~W}$ & \\
\hline & $\begin{array}{l}\text { Total power incl. } \\
\text { maturity margin }\end{array}$ & & $608.3 \mathrm{~W}$ & \\
\hline & Power harness losses & & $12.2 \mathrm{~W}$ & $2 \%$ \\
\hline & $\begin{array}{l}\text { PCDU conversion } \\
\text { losses }\end{array}$ & & $36.5 \mathrm{~W}$ & $6 \%$ \\
\hline & Required system power & & $657.0 \mathrm{~W}$ & \\
\hline & System power margin & & $32.8 \mathrm{~W}$ & $5 \%$ \\
\hline & $\begin{array}{l}\text { Minimum required solar } \\
\text { array output at EOL }\end{array}$ & & $689.8 \mathrm{~W}$ & \\
\hline
\end{tabular}

constraints are due to the test mass capacitive readout sensitivity and optical readout could be used instead, leaving the capacitive system for actuation only.

4.7 Current heritage (assumed bus) and technology readiness level

Already in 1997, a proposal for an ultra-stable atomic clock in space, PHARAO, was submitted to ESA [13] and accepted as the main part of the 'Atomic Clock Ensemble in Space' (ACES) for the Microgravity Application Promotion (MAP). ACES will fly as external payload on the Space Station performing tests in fundamental physics and providing an ultra-high performance global time scale.

The HYPER [14] proposal and assessment study were a further step towards realising the full potential of atom optics in space. The Concurrent Design Facility (CDF) Team in close collaboration with members of the HYPER 
Science Core Team carried out the HYPER assessment study at ESTEC [15]. The study was followed by an Industrial System Level Study conducted by Astrium D (with Astrium Ltd (UK), Galileo Avionica(I), ZARM (D)) in 2001. It was not selected because the technique of cold atoms was not considered mature enough for a space mission.

Several initiatives in European and the US [16, 17] aim to demonstrate the technological feasibility of cold atom sensors. With the launch of the development of a mobile BEC platform project for microgravity experiments in the drop tower and during parabolic flights within a pilot project, running since January 2004, the DLR took a first step to establish this field of research in Germany. The platform, which is called QUANTUS (for QuantengaseunterSchwerelosigkeit) will allow to explore the new field of quantum gases under microgravity. The pilot projects aims for a first technological demonstration of the feasibility of such experiments at the drop tower [18]. For the first time, in 2006, a source, which continuously produces cold atoms, was successfully operated in the drop tower during free fall and serves now for tests purposes [19]. The prospects of such an experiment, however, cover the study of quantum gases in the regime of unperturbed evolution with longest unperturbed time of flight. The research will be performed with regard to scientific and technological aspects, from fundamental physical questions such as measurements of highest precision in atom interferometric set-ups. Worldwide (China, Europe and the US) several activities are launched to investigate cold atom sensors for space applications. Most prominent examples are the ICE project in France [20], exploring ultra-cold matter wave sensors, the ESA MAP project on a cold atom inertial sensors as well as gravigradiometer and gravimeter at JPL and Stanford University.

The MWXG structural design is driven the stability requirements required by the PLM. The residual acceleration requirements on MWXG, while not as demanding as LISA, are demanding and need a precision drag-free attitude control system. The spacecraft structure will be based on LPF. The architecture and the related technology are state-of-the-art with European industry. A lot of effort has been done to develop key components for missions like LPF, LISA or Microscope. Therefore, the technology readiness level is quite advanced. On system level the satellite bus based on LPF has a TRL of 6 . However, due to the LPF launch scheduled for early 2010, the technology will be much more advanced during the subsequent mission phases of MWXG. At the beginning of the B2/C/D phase for MWXG in early 2012 the baselined technology would even already be flight proven.

\section{Key technology areas}

5.1 Payload TRL level and technology development strategy if applicable

Cold atom interferometry on the ground has been under development since the early 1990s [21]. High precision ground-based atom interferometers proved 
their outstanding performance as atomic clocks, as inertial sensors and as gyroscopes. Gravity meters and gravity gradiometers based on cold atoms are competing with state-of-the-art Michelson gravimeters based on falling corner cubes. Even though other limitations reduce this by several orders of magnitude the remaining sensitivity improvement is still impressive [11, 22-28]. Activities have started to realise compact and transportable high-precision inertial sensors in Berlin, in Hannover, in Florence and in Paris. In the last few years major breakthroughs were realised on the ground in the fields of atomic clocks, atom optics, atom interferometry, atom cooling by lasers and Bose-Einstein condensates.

A part of the payload elements are currently tested during drop tower tests and parabolic flights such as QUANTUS (DLR) and ICE (CNES). ESA studies and programmes procure transportable atom interferometers as well as studies of the current limitations of atomic sources.

\section{Atom interferometer}

- Atomic source \& detection:

I) Demonstrate the generation of $10^{10}$ atoms at $1 \mu \mathrm{K}$

II) Demonstrate a signal to noise ratio of 10.000 to 50.000 (current limit 8.000)

III) Demonstrate overlapping of the atomic trajectories with the required targeted accelerational accuracy

- Atom interferometer: Systematic by simultaneous operation of two atom interferometer (interactions, AC Stark shift)

- Development of an adequate ultra-stable oscillator both for Rubidium and Potassium

\section{Laser}

- Lasers for cooling rubidium and potassium, exist as breadboard models, used in drop tower experiments and parabolic flights

- Heritage from PHARAO technology

- Laser for trapping atoms in a dipole trap (either a high power laser or an enhancement cavity)

\subsection{Mission and spacecraft technology challenges}

The mission challenges are very similar to MiCROSCOPE or STEP/GAUGE concerning the satellite control. However, with the successful demonstration of LPF there will no significant remaining mission or spacecraft challenges. The drag-free performance will however need to be reassessed for MWXG in LEO. 
MWXG mission summary

Scientific objectives \& new technologies:

Payload module

Mass

Power

Dimensions

Optical bench

Atom preparation

Bench

Payload subsystems

Mass

Power

Laser bench

Orbit

Launcher

Launch year

Mission lifetime

Spacecraft launch

mass

AOCS

Drag-free

performance

Propulsion

Power

Telemetry

Ground segment
A quantum test of the principle of equivalence:

Comparison the equal rate of free fall of various isotopes of distinct atomic species by using precision atom interferometry in the vicinity of the earth with an accuracy of 1 part of $10^{16}$.

Mapping the spatial structure of the gravitational field

Demonstration of the superior performance of cold atom sensors for drag-free spacecraft control

Accommodated in central cylinder of s/c structure

$135 \mathrm{~kg}$

$51 \mathrm{~W}$ (maximum)

$785 \mathrm{~mm}$ diameter $\times 840 \mathrm{~mm}$ height

Optical elements for coherent atom manipulation

1 drag-free proof mass

2 atom interferometers based on potassium and rubidium accommodated in 2 magnetically shielded vacuum chambers

Optics for atom preparation and detection

Various units accomodated in outer compartments of s/c structure

$139 \mathrm{~kg}$

$184 \mathrm{~W}$

Laser for atom interferometry, preparation (e.g. laser trapping, cooling) and detection of the atoms

High-precision microwave synthesizer as reference for the phase lock of the Raman lasers for coherent transitions of rubidium or potassium

Diode laser based precision source

Acousto-optical modulation and switching

Fiber coupled to physics package

Dawn-dusk, sun-synchronous circular orbit at 1,000 km altitude, $98.48^{\circ}$ inclination

Low-cost Vega launcher; Rockot launcher feasible as back-up

2017 or later

2 years (nominal)

$723.6 \mathrm{~kg}$

Primary AOCS (responsibility of spacecraft) + secondary AOCS (responsibility of Payload Module) for error generation during Science Mode (drag-free control and fine pointing)

$10-10 \mathrm{~g}$ at $0.3 \mathrm{~Hz}$

$16 \times 150 \mu \mathrm{N}$ FEEP thrusters $+8 \times 40 \mathrm{mN}$ cold-gas

thrusters

$690 \mathrm{~W}(\mathrm{EOL})$

Fixed multijunctionGaAs solar array $+6 \mathrm{Ah} \mathrm{Li-ion}$ battery

S-band, $500 \mathrm{kbps}$ during single 12-min pass per day, total of $190 \mathrm{Mbit} / \mathrm{day}$

$15 \mathrm{~m}$-antenna at the ESA Kiruna station, Mission Control from ESOC 
Acknowledgements The QUANTUS-Team acknowledges support from the DLR under the contract 50 WM 0508 and contract DLR 50 WM 0346.

\section{References}

1. Damour, T., Blaser, J.P.: Optimizing the choice of materials in equivalence principle experiments. Proc. Rencontre de Moriond (Villars). Editions Frontières, Gif-sur-Yvette (1993)

2. Eötvös, R.B.v. : Math. Nat. Wiss. Ber. Ung. 8(65), 448 (1891)

3. Eötvös, R.B.v., Pekár, D., Fekete, E.: Ueber die Anziehung der Erde auf verschiedene Substanzen. Ann. Phys. 68, 11 (1922)

4. Roll, P.G., Krotkov, R., Dicke, R.H.: The equivalence of inertial and passive gravitational mass. Ann. Phys. 26, 442 (1964)

5. Braginsky, V.B., Panov, V.I.: The equivalence of inertial and passive gravitational mass. Sov. Phys. JETP 34, 463 (1972)

6. Baessler, S., Heckel, B.R., Adelberger, E.G., Gundlach, J.H., Schmidt, U., Swanson, H.E.: Improved test of the equivalence principle for gravitational self-energy. Phys. Rev. Lett. 83(18), 3585 (1999)

7. Smith, G.L., Hoyle, C.D., Gundlach, J.H., Adelberger, E.G., Heckel, B.R., Swanson, H.E.: Short-range tests of the equivalence principle. Phys. Rev. D 61, 022001 (2000)

8. http://smsc.cnes.fr/MICROSCOPE/

9. Bender, P., Brillet, A., Ciufolini, I., Cruise, A.M., Cutler, C., Danzmann, K., Fidecaro, F., Folkner, W.M., Hough, J., McNamara, P., Peterseim, M., Robertson, D., Rodrigues, M., Rüdiger, A., Sandford, M., Schäfer, G., Schilling, R., Schutz, B., Speake, C., Stebbins, R.T., Sumner, T., Touboul, P., Vinet, J.-Y., Vitale, S., Ward, H., Winkler, W.: LISA, Laser Interferometer Space Antenna, Pre Phase A Report (PPA2), MPQ 233, München (1998)

10. Sneeuw, N., Rummel, R., Müller, J.: The Earth's gravity field from the STEP mission. Class. Quantum Grav. 13, A113-A117 (1996)

11. Peters, A., Chung, K.Y., Chu, S.: Measurement of gravitational acceleration by dropping atoms. Nature 400, 849 (1999)

12. Snadden, M.J., McGuirk, J.M., Bouyer, P., Haritos, K.G., Kasevich, M.A.: Measurement of the earth's gravity gradient with an atom interferometer-based gravity gradiometer. Phys. Rev. Lett. 81, 971 (1998)

13. Laurent, P., Lemonde, P., Simon, E., Santorelli, G., Clairon, A., Dimarcq, N., Petit, P., Audoin, C., Salomon, C.: A cold atom clock in absence of gravity. Eur. Phys. J. D 3, 201 (1998)

14. Jentsch, C., Müller, T., Rasel, E.M., Ertmer, W.: HYPER: a satellite mission in fundamental physics based on high precision atom interferometry. Gen. Relative. Gravit. 36(10), 2197-2221 (2004) (October)

15. Assessment-Study Report, HYPER Hyper-Precision Cold Atom Interferometry in Space, ESA-SCI(2000)10, July 2000

16. Jefferts, S.R., Heavner, T.P., Hollberg, L.W., Kitching, J., Meekhof, D.M., Parker, T.E., Phillips, W., Rolston, S., Robinson, H.G., Shirley, J.H., Sullivan, D.B., Walls, F.L., Ashby, N., Klipstein, W.M., Maleki, L., Seidel, D., Thompson, R., Wu, S., Young, L., Vessot, R.F.C., De Marchi, A.: Joint Meet. Eur. Freq. Time Forum and The IEEE Int. Freq. Contr. Symp., Besançon, France pp. 141-144 (1999) (April)

17. Fertig, C., Gibble, K.: Measurement and cancellation of the cold collision frequency shift in an $87 \mathrm{rb}$ fountain clock. IEEE/EIA Int. Freq. Contr. Symp., Kansas City, MO, pp. 676-679 (2000) (June)

18. Vogel, A., Schmidt, M., Sengstock, K., Bongs, K., Lewoczko, W., Schuldt, T., Peters, A., van Zoest, T., Ertmer, W., Rasel, E., Steinmetz, T., Reichel, J., Könemann, T., Brinkmann, W., Göklü, E., Lämmerzahl, C., Dittus, H.J., Nandi, G., Schleich, W.P., Walser, R.: Bose-Einstein condensates in microgravity. Appl. Phys. B 84, 663 (2006)

19. Könemann, T., et al.: A freely falling magneto-optical trap drop tower experiment. Appl. Phys. B 84, 431-438 (2006)

20. Nyman, R.A., et al.: I.C.E.: a transportable atomic inertial sensor for test in microgravity. Appl. Phys. B 84, 673-681 (2006) 
21. Bermann, P.R. (ed.): Atom Interferometry. Academic, Boston, MA (1997)

22. Wicht, A., Hensley, J.M., Sarajlic, E., Chu, S.: A preliminary measurement of the fine structure constant based on atom interferometry. Physica Scripta. T102, 82 (2002)

23. Bertoldi, A., Lamporesi, G., Cacciapuoti, L., deAngelis, M., Fattori, M., Petelski, T., Peters, A., Prevedelli, M., Stuhler, J., Tino, G.M.: Atom interferometry gravity-gradiometer for the determination of the Newtonian gravitational constant G. Eur. Phys. J. D 40, 271 (2006)

24. Lamporesi, G., Bertoldi, A., Cacciapuoti, L., Prevedelli, M., Tino, G.M.: Determination of the Newtonian gravitational constant using atom interferometry. PRL 100, 050801 (2008)

25. Fixler, J.B., Foster, G.T., McGuirk, J.M., Kasevich, M.A.: Atom interferometer measurement of the newtonian constant of gravity. Science. 315, 74 (2007)

26. Gustavson, T.L., Bouyer, P., Kasevich, M.A.: Precision rotation measurements with an atom interferometer gyroscope. Phys. Rev. Lett. 78, 2046 (1997)

27. Kasevich, M.A.: Coherence with atoms. Science 298, 1363 (2002)

28. Dimopoulos, S., Graham, P.W., Hogan, J.M., Kasevich, M.A.: Testing general relativity with atom interferometry. Phys. Rev. Lett. 98, 111102 (2007)

29. Will, C.M.: The confrontation between general relativity and experiment. http://relativity. livingreviews.org/Articles/lrr-2006-3/ 